\title{
Identity and Aboutness
}

\author{
Benjamin Brast-McKie ${ }^{1}$
}

Received: 5 May 2020 / Accepted: 10 February 2021 / Published online: 25 October 2021

(C) The Author(s) 2021

\begin{abstract}
This paper develops a theory of propositional identity which distinguishes necessarily equivalent propositions that differ in subject-matter. Rather than forming a Boolean lattice as in extensional and intensional semantic theories, the space of propositions forms a non-interlaced bilattice. After motivating a departure from tradition by way of a number of plausible principles for subject-matter, I will provide a Finean state semantics for a novel theory of propositions, presenting arguments against the convexity and nonvacuity constraints which Fine (Journal of Philosophical Logic, 4545, 199-226 2016, 2017a, b) introduces. I will then move to compare the resulting logic of propositional identity ( $\mathrm{PI}^{1}$ ) with Correia's (The Review of Symbolic Logic, 9, 103122 2016) logic of generalised identity (GI), as well as the first degree fragment of Angell's (1989) logic of analytic containment (AC). The paper concludes by extending $\mathrm{PI}^{1}$ to include axioms and rules for a subject-matter operator, providing a much broader theory of subject-matter than the principles with which I will begin.
\end{abstract}

Keywords Identity $\cdot$ Subject-matter $\cdot$ Hyperintensionality $\cdot$ State semantics

\section{Intensionalism}

When do two sentences express the same proposition in virtue of their logical form? It is important to stress that the present understanding of a proposition is intended to be worldly: propositions are, so to speak, things being a certain way rather than representations of things being some way or other. Intensionalism is the view that what it is for propositions to be identical is for them to be necessarily equivalent. For instance, reading ${ }^{\ulcorner} \varphi \equiv \psi{ }^{\urcorner}$as ${ }^{\ulcorner}$For it to be the case that $\varphi$ just is for it to be the case that $\psi^{\urcorner}$, Rayo [36, p. 66] writes, “' $\phi \equiv \psi$ ' should be thought of as equivalent

I am greatly indebted to Mathias Böhm, Kit Fine, James Kirkpatrick, Ofra Magidor, Michail Peramatzis, James Studd, and Tim Williamson for helpful comments and discussion.

Benjamin Brast-McKie

benbrastmckie@gmail.com

1 University of Oxford, Oxford, England 
to " $\square(\phi \leftrightarrow \psi)$ '”. Part of what makes intensionalism appealing is that a simple and strong theory of necessary equivalence may be derived in any normal modal logic, shedding light on the nature of propositional identity and the abstract structure of the space of propositions. Intensionalism is to be contrasted with the view that necessary equivalence approximates propositional identity despite failing to be co-extensive with propositional identity, or else given a specific application, that necessary equivalence may prove to be of some utility. Certainly it should be admitted that intensional theories of propositions have been of great utility within philosophy, logic, linguistics, and computer science. Nevertheless, the present inquiry concerns the nature of propositional identity itself, and not some approximation or useful application.

Instead of defining propositional identity as the intensionalist does, or in yet some further way, primitivism claims that propositional identity is conceptually basic, and so an informative definition in other terms cannot be provided. Accordingly, primitivists cannot follow intensionalists in employing a modal logic to derive a range of theorems for propositional identity from its definition. In an attempt to characterise propositional identity, a primitivist is forced to make a fresh start by axiomatising propositional identity rather than defining propositional identity in terms of other primitive notions. Of course, free from all constraints, little progress can be made, leaving one with no more than suspicions about which identities hold in full generality. For instance, is $A$ the same or different proposition as either $A \wedge A$ or $A \vee A$ ? What about $A$ and either of the propositions $A \vee(A \wedge B)$ or $A \wedge(A \vee B)$ ?

Even if propositional identity cannot be defined in other terms, a primitivist must nevertheless look to some prior conception of the theoretical role that propositions are meant to play in order to guide the ambition to axiomatise propositional identity. For instance, suppose that: (1) the proposition expressed by a sentence on a given interpretation is identified with that sentence's truth-condition; where (2) the truthcondition for an interpreted sentence is taken to be the set of possible worlds in which that sentence is true. ${ }^{1}$ Leaving the interpretation implicit, it follows that sentences which are true in the same possible worlds express the same proposition, where of course sentences which express the same proposition are true in the same possible worlds, and so propositional identity ends up co-extensive with necessary equivalence. In particular, ' ${ }^{\top} A^{\urcorner}$is true in the same worlds as ${ }^{\ulcorner} A \vee(A \wedge B)^{\urcorner}$, and so given (1) and (2), the propositions expressed by ${ }^{\ulcorner} A{ }^{\urcorner}$and ${ }^{\top} A \vee(A \wedge B)^{\urcorner}$are identical, and so $A \equiv A \vee(A \wedge B) .{ }^{2}$ Insofar as the theoretical role of propositional identity does not require more than sameness in modal profile of the interpreted sentences flanking the propositional identity sign, such conclusions are easy to accept. However, it is natural to object that although ${ }^{\ulcorner} A^{\urcorner}$and ${ }^{\ulcorner} A \vee(A \wedge B)^{\urcorner}$are true in the same possible worlds, these sentences may fail to have the same subject-matter, where the subject-matter of an interpreted sentence is what that interpreted sentence is about. For instance, on its intended interpretation, the sentence 'It is raining, or both raining and snowing' is partly about it snowing, where the same cannot be said of the sentence 'It is raining'. Without giving up on a truth-conditional account of interpreted sentences as in (1),

\footnotetext{
${ }^{1}$ Or one could take truth-conditions to be characteristic functions from worlds to truth-values.

${ }^{2}$ I will mostly use upper-case Roman letters to express propositions, relying on context to resolve usemention ambiguities, while occasionally employing corner quotes for clarity.
} 
we may seek to refine our conception of a sentence's truth-condition, replacing (2) with some alternative, guided by the aim to accommodate sameness in subject-matter of the sentences flanking a propositional identity sign.

Setting aside whether primitivism is the right view of propositional identity or not, I will take the propositional identity operator ' $\equiv$ ' to be a primitive term for the purposes of this paper. Instead of offering a definition, the present aim will be to present a logic of propositional identity which is both motivated and constrained by the ambition to respect sameness of subject-matter in addition to necessary equivalence. By distinguishing necessarily equivalent propositions which differ in subject-matter, the theory of propositions developed below will provide novel theoretical resources. Rather than exploring any particular application of these resources, I will be concerned to trace the contours of the resulting hyperintensional theory of propositions by providing a definition of logical consequence as well as a derivability relation for the first-degree fragment of a language for propositional identity.

It is worth contrasting an opposing strategy in which an intensional theory of propositions is merely augmented with a theory of subject-matter. For instance, assuming propositions to be sets of possible worlds, Lewis [32] identifies subjectmatters with partitions of the set of all worlds, writing:

A proposition is about a subject matter, and it is a subject matter of the proposition, iff the truth value of that proposition supervenes on that subject matter.

[...] When we think of subject matters as partitions, we can say that $P$ is about $M$ iff each cell of $M$ either implies or contradicts $P$.

The cells of a partition can be thought of as the different ways for the proposition in question to be true or false, where $M$ includes $N$ just in case every cell of $N$ is a union of cells of $M .{ }^{3}$ For instance, consider the proposition $P_{1}$ that there are more than a hundred stars. One such subject-matter of $P_{1}$ is the partition $M_{1}$ which consists of $P_{1}$ and its complement within the set of all worlds $W$, whereas another partition $M_{2}$ groups worlds together into cells which have the same number of stars. Accordingly, $M_{2}$ includes $M_{1}$. Not only is there no unique subject-matter which each proposition is about, Lewis [32, p. 171-2] admits that non-contingent propositions are about every subject-matter, "since there is no way at all for two worlds to give it different truth values, a fortiori there is no way for two worlds to give it different truth values without differing with respect to the subject matter". 4 Thus 'The gold atom $\alpha$ has 79 protons', ' $2+2=5$ ', and all instances of ' $A \vee \neg A^{\top}$ and ' $B \wedge \neg B$ ' express propositions which, according to Lewis, are about all subject-matters. Additionally, by identifying necessarily equivalent propositions, Lewis makes all necessarily equivalent propositions about the same subject-matters. For instance, $A \vee(A \wedge B), A \wedge(A \vee B)$, and $A$ will have the same subject-matters for any $A$ and $B$, and so Lewis cannot accommodate the apparent difference in subject-matter between the proposition that it is raining and the proposition that it is raining or both raining and snowing.

\footnotetext{
${ }^{3}$ Lewis [33] speaks of inclusion, though it is more natural to read ${ }^{\ulcorner} M$ includes $N^{\urcorner}$as ${ }^{\ulcorner} M$ refines $N^{\urcorner}$.

${ }^{4}$ Lewis [32, p. 164] does introduce the concept of least subject-matters, but admits that there need not always be a least subject-matter for a given proposition.
} 
Abstracting from the details of Lewis' account, the broader strategy aims to capture differences in subject-matter while maintaining an intensional theory of propositions by asking: for which values of $X$ does the product $\mathcal{P}(W) \times X$ draw enough distinctions to encode differences in subject-matter $?^{5}$ As fruitful as intensional theories of propositions have been for many applications, no such account can accommodate differences in subject-matter between necessarily equivalent propositions. However, there is no need to accept this limitation. As Perry [35] writes:

[T] he problem of necessary equivalent propositions is simply a fly bottle that did not have to be flown into. The solution is to fly out, not to argue that, all things considered, maybe it is not such a bad bottle to be in.

(p. 191)

Rather than attempting either to maintain an intensional theory of propositions, or else to augment an intensional theory of propositions with a theory of subject-matters, I will defend a hyperintensional theory of propositions which does not presume that the particular form of hyperintensionality in question can or should be factored into intensional and non-intensional components. ${ }^{6}$ Before attempting to provide such an account, it will be important to motivate criteria for an adequate theory of propositional identity. Accordingly, Section 2 will present a number of principles which I will assume that any adequate account of subject-matter ought to include along with a minimal theory of propositional identity, where the adoption of these principles will guide our departure from standard Boolean theories of propositional identity. By drawing on the resources of Kit Fine's state semantics, Section 3 will present a hyperintensional theory of propositions which accommodates differences in subject-matter, comparing the result with Fine's [13-15] theory of regular propositions in Section 4. After presenting a first-degree logic for propositional identity $\left(\mathrm{PI}^{1}\right)$, Section 5 will contrast Correia's [9] logic of generalised identity (GI) which I show has the unwanted consequence of making negation an opaque operator. I will conclude in Section 6 by extending $\mathrm{PI}^{1}$ to include axioms and rules of inference for a subject-matter operator, providing not only a broader theory of subject-matter, but one in which we may derive the first degree fragment of Angell's [2] logic of analytic containment (AC) in addition to comparing Fine's [13, 15-17] account of subjectmatter. In the Appendix, I will present a few formal results which will be of use at various points throughout the paper.

\section{Subject-Matter}

In order to facilitate the presentation of a theory of subject-matter, we may introduce the sentential operator ' $\sigma$ ', where $\sigma A$ is the subject-matter of $A$. Whereas Fine [13,

\footnotetext{
${ }^{5}$ See $[4,27,38]$ for other theories of this kind.

${ }^{6}$ As Perry [35, p. 176] also observes, "the view that language was basically intensional, is older than possible-worlds semantics. Basically, intensions are entities that provide some principle of classification, and that have an identity, independently of the objects so classified". Rather than attempting to uproot the already entrenched practice of taking 'intensions' to be functions from possible-worlds to truth-values, I will maintain the spirit of Perry's critique of restricting attention to a possible worlds understanding of intensions by defending a hyperintensional alternative.
} 
15-17] takes subject-matters to be states-of-affairs, I will maintain a propositional account of subject-matter, whereby the subject-matter of an interpreted sentence is a proposition. ${ }^{7}$ For instance, the subject-matter of 'It is raining' will be a rainy weather proposition. More specifically, Section 6 will argue that there is good reason to read ${ }^{\ulcorner} \sigma A^{\top}$ as 'It is partially the case that $A$ or partially not the case that $A^{\top}$. By letting $A \leftrightarrow B:=\sigma A \equiv \sigma B$, where we may then take ${ }^{\ulcorner} A \leftrightarrow B^{\urcorner}$to have the informal reading ' $A$ and $B$ have the same subject-matter', I will adopt the principles:

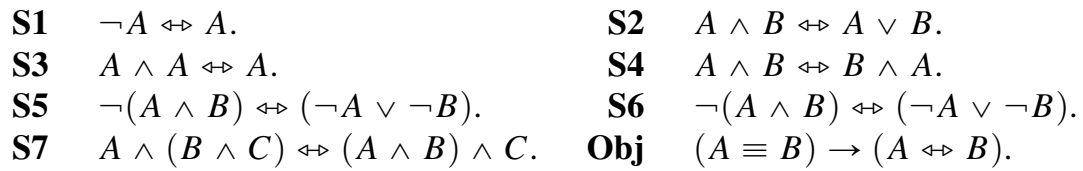

Setting aside differences in formalisation, the principles above are widely accepted. ${ }^{8}$ Given that $\neg A \equiv A$ and $A \wedge B \equiv A \vee B$ do not hold in general, it follows from S1 and $\mathbf{S 2}$ that the converse of $\mathbf{O b j}$ admits of exceptions. Rather, Obj makes sameness of subject-matter a necessary but insufficient condition for propositional identity. Nevertheless, Obj asserts that what an interpreted sentence is about is solely a function of the proposition expressed by that sentence on its interpretation irrespective of the features unique to that sentence, or the concepts by which that proposition happens to be expressed. For instance, given that for Hesperus to be rising just is for Phosphorus to be rising, it follows by Obj that the subject matter of 'Hesperus is rising' is the same as the subject-matter of 'Phosphorus is rising'. In slogan, the present theory takes subject-matter to be insensitive to differences in guise.

I will refer to theories of subject-matter which grant $\mathbf{O b j}$ as objective, and theories which reject Obj as representational. By contrast with objective theories, representational theories make subject-matter at least partially a function of the means by which a given proposition is expressed. For instance, suppose that one were to take 'Hesperus is rising' and 'Phosphorus is rising' to differ in subject-matter despite expressing the same proposition. Insofar as the subject-matter of an interpreted sentence is what that sentence is about, what 'Hesperus is rising' and 'Phosphorus is rising' are about must differ. But this strikes a false note, for both sentences appear to be entirely about the movement of the same astrological body relative to one's position. Since the sentences 'Hesperus is rising' and 'Phosphorus is rising' express the same proposition, the only remaining differences between these sentences are representational in nature, having to do with the means by which each sentence expresses the same proposition. For instance, one might consider the syntactic differences between sentences, or else the different concepts thereby expressed. However, it would be inappropriate to identify the subject-matter of an interpreted sentence with the means by which that sentence expresses the proposition that it does, at least insofar as the subject-matter of

\footnotetext{
${ }^{7}$ Although states will be taken to be objects, one may think of the states in the intended model as propositions, for states are objects which may either obtain or fail to obtain.

${ }^{8}$ Even in adopting an objectual account of subject matter where ${ }^{\top} \sigma A^{\top}$ is taken to be a singular term, the principles above may be maintained by instead defining $A \leftrightarrow B:=\sigma A=\sigma B$. See $[17,27,32,35,38]$ for accounts which are committed to analogues of the principles above.
} 
an interpreted sentence is what that sentence is about. For instance, neither 'Hesperus is rising' nor 'Phosphorus is rising' are about anything the least bit representational, and so the ways in which these sentences differ cannot be identified with their different subject-matters. Nevertheless, a representational theory of subject-matter could take the subject-matter of an interpreted sentence to be a function of the means by which that sentence expresses a proposition. Instead of pursuing this line, I will develop an objective theory of subject-matter where the subject-matter of an interpreted sentence is a function of the proposition which that sentence expresses. Thus I will speak directly of the subject-matter of each proposition, whether or not there is a sentence which expresses that proposition on a given interpretation.

Even in granting Obj, this principle cannot provide positive determinations of which propositional identities hold on account of including the propositional identity sign in its antecedent. Nevertheless, it follows from Obj that discrepancies in the subject-matters of $A$ and $B$ entail that $A$ and $B$ are distinct. In this way, a theory of subject-matter may inform our present aim to provide a theory of propositional identity. We may also draw the following connection between subject-mater and relevance, where ${ }^{\ulcorner} A \leq B^{\urcorner}$reads ' $\mathrm{r}$ t being the case that $A$ is wholly relevant to it being the case that $B^{\urcorner}$, or for ease, ${ }^{\ulcorner} A$ is relevant to $B^{\urcorner}$:

Rel $(A \leftrightarrow B) \rightarrow[(C \leq A) \rightarrow(C \leq B)]$.

If $A$ and $B$ share the same subject-matter- if what $A$ and $B$ are about is the samethen whatever is relevant to $A$ must also be relevant to $B$. Together, Obj and Rel provide a means by which to distinguish propositions, for if $C \leq A$ and $C \nless \subset B$, it follows that $A \leftrightarrow \triangleright B$ by Rel, and so $A \not \equiv B$ by Obj. Of course, such evaluations will depend on judgements about relevance. Nevertheless, Obj and Rel provide an additional basis by which to evaluate propositional identity claims for truth.

Recall the claim from before that although $A$ and $A \vee(A \wedge B)$ have the same modal profile, they may fail to share the same subject-matter. Even more starkly, $A \vee \neg A$ and $B \vee \neg B$ are both necessary but may diverge completely in subject-matter. For instance, the sentences 'I am sitting or not sitting' and 'Grass is green or not green' both express necessary propositions despite having entirely distinct subject-matters, where neither is even partially about the other. In order to account for the possible divergence in subject-matter between $A \vee \neg A$ and $B \vee \neg B$, we may observe that even without giving a full theory of relevance, it is natural to accept the following:
L1 $A \leq A \vee B$.
L2 $A \leq A \wedge B$.
L3 $B \leq A \vee B$.
L4 $B \leq A \wedge B$.

Given some $A$ and $B$ for which $A \nless B \vee \neg B$, we know by L1 that $A \preceq A \vee \neg A$, and so $(A \vee \neg A) \not \equiv(B \vee \neg B)$ follows by Obj and Rel, where a similar argument concludes that $(A \wedge \neg A) \not \equiv(B \wedge \neg B)$ on the basis of L2. ${ }^{9}$ For instance, my sitting fails to be wholly relevant to grass being green or not green, though of course my

\footnotetext{
${ }^{9}$ Given the definition of relevance in Section 6, we may show that $A \leq B \vee \neg B$ just in case $A \leq B$.
} 
sitting is relevant to me sitting or not sitting. Thus by Obj and Rel, it is not the case that for me to be sitting or not sitting just is for grass to be green or not green.

We may provide similar arguments against the absorption laws. Given some $A$ and $B$ where $B \nless A$ but $B \preceq[A \vee(A \wedge B)]$, it follows by Obj and Rel that $A \not \equiv A \vee(A \wedge B) .{ }^{10}$ For instance, although it snowing is relevant to it raining or both raining and snowing, it snowing fails to be relevant to it raining, and so by Obj and Rel, for it to be raining is not for it to be raining or both raining and snowing. An analogous argument shows that not all instances of $A \equiv A \wedge(A \vee B)$ hold. For these reasons, I will take exception to the following Boolean identities:

$\begin{array}{llll}\text { \#Necs } & (A \vee \neg A) \equiv(B \vee \neg B) . & \text { \#Abs1 } & A \equiv A \vee(A \wedge B) . \\ \text { \#Imps } & (A \wedge \neg A) \equiv(B \wedge \neg B) . & \text { \#Abs2 } & A \equiv A \wedge(A \vee B) .\end{array}$

Whereas intensional theories of propositions are Boolean insofar as they affirm all of the Boolean identities, the present paper will provide a non-Boolean alternative. Nevertheless, in accordance with a conservative methodology, I will maintain as many of the Boolean identities as possible without ignoring differences in subject-matter. More specifically, I will assume that just as differences in either the subject-matter or the modal profile of $A$ and $B$ provide a reason to distinguish $A$ and $B$, sameness in both the subject-matter and the modal profile of $A$ and $B$ provides at least a defeasible reason to maintain the identity of $A$ and $B$.

In order to begin to evaluate the broader space of propositional identities, I will assume that anything deserving of the title 'propositional identity' ought to satisfy all instances of the following core principles:

$$
\begin{array}{lll}
\text { Ref } \quad A \equiv A . & \text { Trans } & (A \equiv B) \rightarrow[(B \equiv C) \rightarrow(A \equiv C)] . \\
\text { Sym }(A \equiv B) \rightarrow(B \equiv A) . & \text { Imp } & (A \equiv B) \rightarrow(A \rightarrow B) .
\end{array}
$$

Rejecting any of the core principles would be to change the topic from propositional identity to something else entirely. Additionally, given the present concern with worldly propositions, I will restrict attention to propositional operators which are insensitive to guise. More precisely, we may say that an operator $\mathcal{Q}$ is transparent in a language $\mathcal{L}$ just in case all instances of the following principle hold, where ${ }^{\top} \vec{O}_{(B / A)}{ }^{\top}$ is the result of freely substituting ${ }^{\ulcorner} B^{\urcorner}$for ${ }^{\ulcorner} A^{\urcorner}$in the sequence of $\mathcal{Q}$ 's operands ${ }^{\ulcorner} \vec{O}^{\urcorner}$:

$$
\text { Func }(A \equiv B) \rightarrow\left[\mathcal{Q}(\vec{O}) \equiv \mathcal{Q}\left(\vec{O}_{(B / A)}\right)\right] .
$$

Intuitively, a sentential operator is transparent in a language just in case it expresses a propositional function, where the output of the function is determined solely by the inputs independent of the means by which those inputs are expressed. A language $\mathcal{L}$ is transparent just in case every operator $\mathcal{Q}$ in $\mathcal{L}$ is transparent in $\mathcal{L}$.

Given that the present concern is with the structure of the space of propositions independent of the structure of the different means of representing those propositions, I will restrict consideration to transparent languages throughout. As P2 in the Appendix shows, any propositional language $\mathcal{L}$ which includes the extensional

\footnotetext{
${ }^{10}$ Both $B \leq[A \vee(A \wedge B)]$ and its dual are theorems of $\mathrm{PI}_{\sigma}^{1}$ presented in Section 6 .
} 
connectives ' $\neg$ ', ' $\wedge$ ', and ' $\vee$ ' along with the propositional identity operator ' $\equiv$ ' is transparent just in case the following principle holds without exception in $\mathcal{L}$, where ${ }^{\ulcorner} C_{(B / A)}{ }^{\top}$ is the result of freely substituting $\left.{ }^{\ulcorner} B\right\urcorner$ for $\left.{ }^{\ulcorner} A\right\urcorner$ anywhere in ${ }^{\ulcorner} C^{\urcorner}$:

$$
\mathbf{L L} \quad(A \equiv B) \rightarrow\left(C \rightarrow C_{(B / A)}\right) .
$$

The principle above expresses Leibniz's law of the indiscernibility of identicals where identicals are required to satisfy the same conditions. Rather than adopting LL as an independent assumption, P2 in the Appendix shows that so long as Ref and Imps hold without exception, $\mathbf{L} \mathbf{L}$ follows from the stipulation that $\mathcal{L}$ is transparent. Since the restriction to transparent languages was motivated by the concern to study the structure of the space of worldly propositions independent of any representational difference in thought or language, LL may be taken to inherit the same motivation given the ambition to provide a theory of identity for worldly propositions.

It is important to stress that in articulating a theory of propositional identity, we need only take a stand on the transparency of a limited range of operators. In particular, I will take the operators for conjunction, disjunction, negation, and subject-matter to be transparent. Letting an operator be opaque in $\mathcal{L}$ just in case it is not transparent in $\mathcal{L}$, the present paper need not take a stand on whether there are genuine cases of opacity, though I take it that there are such genuine cases, where it is in virtue of this fact that synonymy is much more fine-grained than propositional identity. ${ }^{11}$

Given that all instances of $\mathbf{L} \mathbf{L}$ hold without exception in a language with operators for conjunction, disjunction, negation, and subject-matter, we may derive S3 - S7 from the following identities by means of classical reasoning:
A1 $A \wedge A \equiv A$.
A2 $A \vee A \equiv A$.
A3 $A \wedge B \equiv B \wedge A$.
A4 $A \vee B \equiv B \vee A$.
A5 $(A \wedge B) \wedge C \equiv A \wedge(B \wedge C)$. A6 $(A \vee B) \vee C \equiv A \vee(B \vee C)$.
A7 $\neg(A \wedge B) \equiv(\neg A \vee \neg B)$.
A8 $\neg(A \vee B) \equiv(\neg A \wedge \neg B)$.
A9 $A \equiv \neg \neg A$.

Given S1 - S7, the identities above respect sameness of subject-matter. Since these identities also respect necessary equivalence, a conservative methodology recommends adopting these principles in the absence of countervailing considerations. Suppose that one were to attempt a similar defence for the following principles:

$$
\begin{array}{ll}
\text { \#Dist1 } & A \vee(B \wedge C) \equiv(A \vee B) \wedge(A \vee C) \\
\text { \#Dist2 } & A \wedge(B \vee C) \equiv(A \wedge B) \vee(A \wedge C)
\end{array}
$$

The principles for subject-matter considered so far do not provide any means by which to evaluate whether the identities above respect sameness of subject-matter. Since intuitive judgements about which principles for subject-matter hold without exception can only carry us so far, the following section will present a semantics for a propositional language, providing a systematic means by which to survey the space of all possible counterexamples to propositional identity claims. Section 4 will then

\footnotetext{
${ }^{11}$ See $[3,3,11]$, and [7] for recent discussion of opacity, as well as Section 6 for further comparison between synonymy and propositional identity.
} 
draw upon this semantics to show that there are strong abductive reasons to exclude \#Dist1 and \#Dist2 from the logic of propositional identity on account of admitting a compelling class of counterexamples.

\section{State Semantics}

In giving up the Boolean identities \#Necs, \#Imps, \#Abs1, and \#Abs2, it remains to provide an alternative non-Boolean theory of propositions. Instead of attempting to axiomatise propositional identity by means of intuition alone, this section will draw on Kit Fine's state semantics in order to provide a theory of propositions which is sensitive to hyperintensional differences in subject-matter, while nevertheless accommodating the identity principles maintained above.

For simplicity, I will focus on the first-degree fragment of a propositional language $\mathcal{L}$ with the sentence letters $\mathbb{L}=\left\{p_{i}: i \in \mathbb{N}\right\}$ and primitive operators $\neg, \vee, \wedge$, and $\equiv$, postponing consideration of extensions which include ' $\sigma$ ' to Section 6 . Given $\mathcal{L}$, we may define the extensional sentences of $\mathcal{L}$ as follows, where $p \in \mathbb{L}$ :

$$
A::=p|\neg A| A \wedge A \mid A \vee A .
$$

Letting $\operatorname{ext}(\mathbb{L})$ be the set of extensional sentences in $\mathcal{L}$, I will take $A \equiv B$ to be an identity sentence in $\mathcal{L}$ for any $A, B \in \operatorname{ext}(\mathbb{L})$, where $\mathrm{id}(\mathbb{L})$ is the set of identity sentences in $\mathcal{L}$. Following Fine [14-16], we may take a state space $\mathcal{S}=\langle S$, $\rangle$ to be any nonempty complete lattice which is defined in the usual way:

Upper Bound: $s \in S$ is an upper bound of $X \subseteq S$ iff $x \sqsubseteq s$ for every $x \in X$.

Least Upper Bound: $s$ is a least upper bound of $X \subseteq S$ iff $s$ is an upper bound of $X$, and $s \sqsubseteq y$ for every upper bound $y$ of $X$.

Complete Lattice: $\mathcal{S}$ is a complete lattice iff every $X \subseteq S$ has a least upper bound.

As the uniqueness of a least upper bound for any set $X$ is readily established, we may refer to the least upper bound $\bigsqcup X$ of $X$ as the fusion of the states in $X$, designating the full state by $\bigsqcup S=$ - and the null state by $\bigsqcup \varnothing=\square$. It remains to employ these resources in order to interpret the identity sentences in $i d(\mathbb{L})$ in a manner which accords with the range of principles defended above.

Given a state space $\mathcal{S}=\langle S, \sqsubseteq\rangle$, I will follow Fine [14, p. 629] in taking propositions to be ordered pairs of sets of states which satisfy some number of constraints depending on the application. Whereas the following section will raise general problems for the theory of regular propositions which Fine [13, 14] develops, the present section will present a competing alternative which I will argue is well suited to the application at hand. In particular, consider the space of normal propositions which may be defined over any state space $\mathcal{S}$ as follows:

Normal Contents: $\mathbb{C}_{\mathcal{S}}=\{X \subseteq S: \bigsqcup Y \in X$ for all nonempty $Y \subseteq X\}$.

Normal Propositions: $\mathbb{P}_{\mathcal{S}}=\left\{\langle X, Y\rangle: X, Y \in \mathbb{C}_{\mathcal{S}}\right\}$. 
An S-model of $\mathcal{L}$ is any ordered triple $M=\langle S, \sqsubseteq,|\cdot|\rangle$ where $S=\langle S$, $\rangle$ is a state space and $|p|=\left\langle|p|^{+},|p|^{-}\right\rangle$with $|p|^{ \pm} \subseteq S$ for every $p \in \mathbb{L} .^{12}$ An $\mathcal{S}$-model $\mathcal{M}$ is normal just in case $|p| \in \mathbb{P}_{\mathcal{S}}$ for every $p \in \mathbb{L}$, where I will take $\mathcal{N}$ to be the class of all normal $\mathcal{S}$-models of $\mathcal{L}$ for any state space $\mathcal{S} .{ }^{13}$

In order to identify which sentences are true in all normal models of $\mathcal{L}$, we must provide semantic clauses for the primitive operators in $\mathcal{L}$. Following Fine [13, 16], I will define exact verification $\Vdash$ and exact falsification $-\|$ by means of the inclusive semantics given below, letting $t . d:=\bigsqcup\{t, d\}$ for ease of exposition:

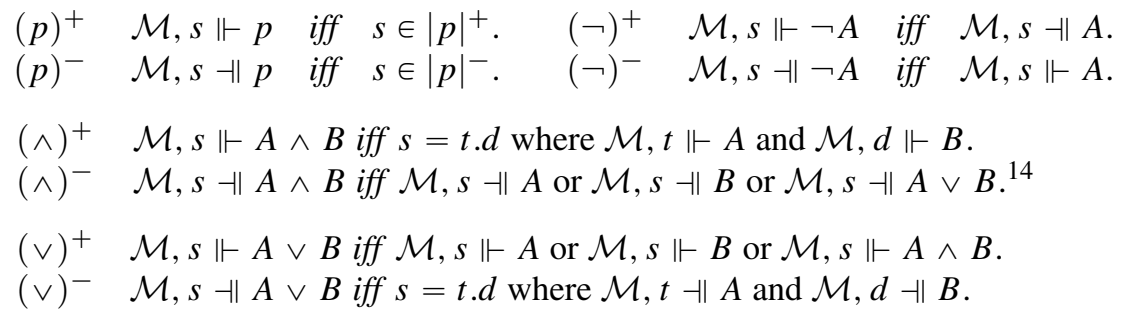

As a useful heuristic, we may consider an intended state space $\mathcal{S}_{I}$, where $S_{I}$ is the set of all states-of-affairs, and $\sqsubseteq_{I}$ is a parthood relation. Instead of considering which sentences are true or false in which possible worlds, where worlds contain a great number of things which are irrelevant to any given sentence, exact verification and falsification is a matter of which sentences are "made" true or false by which states, where the truth-makers and falsity-makers must be wholly relevant to the sentences that they make true or false. ${ }^{15}$ Although the exact verifiers for a conjunction are determined by the exact verifiers for its conjuncts, and the exact verifiers for a disjunction are determined by the exact verifiers for its disjuncts, the same cannot be said for negation. ${ }^{16}$ In particular, one cannot take any state which does not exactly verify a sentence to exactly verify its negation, at least insofar as states are required to be wholly relevant to the sentences which they exactly verify or falsify. It is for this reason that the state semantics assumes a bilateral form, extending consideration to exact falsifiers in addition to exact verifiers so as to identify the exact verifiers (falsifiers) for a sentence with the exact falsifiers (verifiers) for the negation of that sentence. In this respect, the inclusive semantics makes an important addition to (1) given in Section 1 by including falsity-conditions alongside truth-conditions.

Constructing the state semantics around the idea that states are required to be wholly relevant to the sentences which they exactly verify or falsify makes the

\footnotetext{
${ }^{12}$ Were one to add top and bottom elements to the language, $\mathcal{S}$-models must assign $|\top|=\langle S, \varnothing\rangle$ and $|\perp|=\langle\varnothing,\{\square\}\rangle$ which are the top and bottom elements with respect to $\leqslant$, where we may let $\top:=\neg \top$ and $\perp:=\neg \perp$ be the top and bottom elements with respect to $\sqsubseteq$. By contrast, [14, p. 630] takes $\langle S,\{\mathbf{-}\}\rangle$ to be a top element on account of excluding consideration of all vacuous propositions. See also Section 4 . ${ }^{13}$ I will employ set notation for convenience, assuming a no-class theory of classes.

${ }^{14}$ Removing the final disjunct from $(\wedge)^{-}$and $(\vee)^{+}$yields the non-inclusive semantics.

${ }^{15}$ See Fine [14-16] for related discussion, as well as Section 4 below.

${ }^{16}$ Instead of considering what is relevant to the truth (falsity) of a sentence, one may consider what is relevant to either the truth or falsity of a sentence. See Section 6 for related discussion.
} 
notions of exact verification and falsification non-monotonic. Focusing on exact verification, Fine [14] brings this point out as follows:

For it is to be a general requirement on verification that a verifier should be relevant as a whole to the statement that it verifies; and in extending a verifier with additional material, this holistic relevance of the verifier to the statement may be lost.

(p. 626)

For instance, if the state $t$ of Julieta thinking exactly verifies the sentence 'Julieta is thinking', and the state $d$ of Julieta writing exactly verifies the sentence 'Julieta is writing', then the fusion $t . d$ fails to be wholly relevant to 'Julieta is thinking' as well as to 'Julieta is writing' on account of including something irrelevant in each case. Nevertheless, the fusion state $t . d$ exactly verifies the conjunction 'Julieta is thinking and writing' on account of being a fusion of exact verifiers for each of its conjuncts, where similarly, any fusion of exact falsifiers for 'Julieta is thinking' and 'Julieta is writing' will exactly falsify the disjunction 'Julieta is thinking or writing'.

It remains to consider the exact verification clause for disjunction, and the exact falsification clause for conjunction. First we may observe that a disjunction is exactly verified by the exact verifiers for either of its disjuncts, and similarly, a conjunction is exactly falsified by the exact falsifiers for either of its conjuncts. Additionally, the inclusive semantics respects the claims that: $(\mathrm{T})$ any exact verifier for a conjunction $A \wedge B$ will also be an exact verifier for the disjunction $A \vee B$; and, (F) any exact falsifier for $A \vee B$ will also be an exact falsifier for $A \wedge B$. In order to justify these latter additions, consider the following property:

Uniformity: A class $\mathcal{K}$ of models of $\mathcal{L}$ is uniform iff for any $\mathcal{S}$-model $\mathcal{M} \in \mathcal{K}$ and $A \in \operatorname{ext}(\mathbb{L})$, there is an $\mathcal{S}$-model $\mathcal{M}_{\star} \in \mathcal{K}$ and $p \in \mathbb{L}$ where $|p|_{\star}^{+}=\{s \in \mathcal{M}: \mathcal{M}, s \Vdash A\}$ and $|p|_{\star}^{-}=\{s \in \mathcal{M}: \mathcal{M}, s \dashv A\}$.

It is natural to require the class of models over which $\mathcal{L}$ is to be interpreted to be uniform since nothing about the sentence letters in $\mathbb{L}$ should prevent them from expressing the same propositions expressed by the complex sentences in $\operatorname{ext}(\mathbb{L})$. Without requiring uniformity to hold, the law of uniform substitution is liable to fail, where uniform substitution is a natural desideratum for any logic. However, were one to give up $(\mathrm{T})$, then the exact verifiers for a disjunction might fail to be closed under fusion, where the same may be said of the exact falsifiers for a conjunction were one to give up (F). ${ }^{17}$ Since the exact verifiers and falsifiers for any sentence letter must be closed under fusion, rejecting either (T) or (F) leads to the non-uniformity of $\mathcal{N}$, thereby providing a reason to maintain the inclusive semantics.

Insofar as we are to restrict consideration to the class of normal models $\mathcal{N}$ of $\mathcal{L}$, uniformity provides a strong reason to maintain the inclusive semantics. In order to motivate the initial restriction to $\mathcal{N}$, it will help to set $|A|^{+}=\{s \in S: \mathcal{M}, s \Vdash A\}$ and $|A|^{-}=\{s \in S: \mathcal{M}, s \dashv A\}$, adopting $|A|=\left\langle|A|^{+},|A|^{-}\right\rangle$as standard notation for the proposition that $A$ expresses in $\mathcal{M}$. We may then provide the following semantic clause for the first-degree identity sentences in $\mathrm{id}(\mathbb{L})$ along with definitions for logical consequence and validity over an arbitrary class of models $\mathcal{K}$ :

\footnotetext{
${ }^{17}$ Fine [13, p. 206] establishes this result in Lemma 6.
} 
$(\equiv) \mathcal{M} \models A \equiv B$ iff $|A|=|B|$.

Logical Consequence: $\varphi \in \operatorname{id}(\mathbb{L})$ is a $\mathcal{K}$-logical consequence of $\Gamma \subseteq \operatorname{id}(\mathbb{L})$, i.e. $\Gamma \models \mathcal{K} \varphi$, just in case for any $\mathcal{M} \in \mathcal{K}$, if $\mathcal{M} \models \gamma$ for all $\gamma \in \Gamma$, then $\mathcal{M} \models \varphi$.

Validity: $\varphi$ is $\mathcal{K}$-valid just in case $\models_{\mathcal{K}} \varphi$.

Suppose that instead of adopting the inclusive semantics, one were to give up (T) and (F), maintaining uniformity by also giving up the closure condition on the sets of states which make up propositions. As Fine [16, p. 563] observes, $A$ and $A \wedge A$ may then diverge in their exact verifiers, where $A$ and $A \vee A$ may diverge in their exact falsifiers, thereby producing counterexamples to A1 and A2. However, since $A$ agrees in both subject-matter and modal profile with $A \wedge A$ and $A \vee A$, we find no reason to distinguish between $A$ and either $A \wedge A$ or $A \vee A$ given our present aims. Thus if the semantics is to validate $\mathbf{A 1}$ and $\mathbf{A 2}$, then $A \wedge A$ and $A \vee A$ must have the same exact verifiers and falsifiers as $A$. It follows that the sets of exact verifiers and falsifiers for $A$ must both be closed under finite fusion in $\mathcal{S}$, where a set of states $X$ is closed under finite fusion in $\mathcal{S}$ just in case $t . d \in X$ whenever both $t, d \in X$.

Let a finite fusion model $\mathcal{M}$ of $\mathcal{L}$ be any $\mathcal{S}$-model of $\mathcal{L}$ where both $|p|^{ \pm}$are closed under finite fusion in $\mathcal{S}$. Since there may be more than one exact verifier or falsifier for a sentence in a finite fusion model of $\mathcal{L}$, it follows that the exact verifiers and falsifiers for a sentence may contain more than what is strictly required to make that sentence true or false respectively, and so may fail to be minimal. ${ }^{18}$ For instance, any fusion of two or more exact verifiers for $A$ is also an exact verifier for $A$ which contains proper parts which exactly verify $A$, where something similar may be said for a fusion of two or more exact falsifiers for $A$. Given that any amount of overdetermination is to be permitted, it is not clear what would motivate a restriction on overdetermination to merely finite fusions. For instance, given a real number $625 \leqslant x \leqslant 740$, we may take $s_{x}$ to be the state of $\alpha$ reflecting light with wavelength $x$ nanometers, where $R=\left\{s_{x}: 625 \leqslant x \leqslant 740\right\}$. Insofar as each $s_{x} \in R$ is an exact verifier for the sentence ' $\alpha$ reflects red light', we may admit that $\bigsqcup Y$ is also an exact verifier for any nonempty $Y \subseteq R .{ }^{19}$ In order for infinite fusions of exact verifiers (falsifiers) to be admitted as exact verifiers (falsifiers) for sentences, I have taken normal propositions to include sets of exact verifier and falsifier states which are closed under infinite rather than finite fusion, restricting attention to the class of models $\mathcal{N}$ in which sentence letters are assigned to normal propositions. We may then show by a simple induction argument that closure under infinite fusion extends to all extensional sentences in $\mathbb{L}$.

Although I will not provide much of an exploration of the modalised state spaces introduced by Fine [16], it is nevertheless worth considering a primitive distinction

\footnotetext{
${ }^{18}$ Compare minimal situations as used by [29] and [28] among others, as well as exemplifiers which [30, 31] later employs.

${ }^{19}$ Considerations of gunk also motivate one to take fusions of sets of exact verifiers of any non-zero cardinality to be exact verifiers. If a gunky ice cube is entirely pink, then the state of any part of it being pink will exactly verify the sentence 'Part of the ice cube is pink', where if $a$ is a part of $b$, then the state of $a$ being pink is also part of the state of $b$ being pink. It follows that for any non-null part of the ice cube $a$, the state of $a$ being pink will consist of an infinite fusion of exact verifiers for the sentence 'Part of the ice cube is pink'. See [37] for discussion of a related issue for Kratzer's situation semantics.
} 
between possible and impossible states, where every part of a possible state is also required to be possible. We may then say that two states $t$ and $d$ are compatible just in case their fusion $t . d$ is possible, and incompatible otherwise. For instance, although the state of my sitting and the state of my standing are both possible, their fusion is impossible, making these states incompatible. Insofar as sentences are to admit of incompatible exact verifiers (falsifier), we may observe that far from an obscure artefact of the framework, impossible states do important work in drawing hyperintensional distinctions. In particular, it is natural to assume that no exact verifier $v$ and falsifier $f$ for a single sentence $A$ could ever be compatible. However, it follows that every exact verifier for $A \wedge \neg A$ is a fusion of an exact verifier and falsifier for $A$, and so must therefore be impossible. Nevertheless, we may observe that whenever the exact verifiers and falsifiers for $A$ and $B$ do not share any parts in common, $A \wedge \neg A$ and $B \wedge \neg B$ are exactly verified and falsified by different impossible states, as are $A \vee \neg A$ and $B \vee \neg B$. Thus $A \wedge \neg A$ and $B \wedge \neg B$ may be distinct despite sharing the same modal profile, where the same may be said for $A \vee \neg A$ and $B \vee \neg B$, and so neither \#Imps nor \#Necs are valid over $\mathcal{N}$, as desired.

In addition to including counterexamples to \#Imps and \#Necs, neither \#Abs1 nor \#Abs 2 are $\mathcal{N}$-valid. Consider the model $\mathcal{M}_{A}=\left\langle S_{A}, \subseteq,|\cdot|{ }_{A}\right\rangle$ where we may let $S_{A}=\mathcal{P}(\{a, b, c, d\})$ with $\left|p_{1}\right|_{A}=\langle\{\{a\}\},\{\{b\}\}\rangle$ and $\left|p_{2}\right|_{A}=\langle\{\{c\}\},\{\{d\}\}\rangle$, for pairwise distinct $a, b, c$, and $d$. We may then derive the following identities:

$$
\begin{aligned}
& \left|p_{1} \vee\left(p_{1} \wedge p_{2}\right)\right|_{A}=\langle\{\{a\},\{a, c\}\},\{\{b\},\{b, d\}\}\rangle ; \\
& \left|p_{1} \wedge\left(p_{2} \vee p_{2}\right)\right|_{A}=\langle\{\{a\},\{a, c\}\},\{\{b\},\{b, d\}\}\rangle .
\end{aligned}
$$

Since $\{\{a\}\} \neq\{\{a\},\{a, c\}\}$ and $\{\{b\}\} \neq\{\{b\},\{b, d\}\}$, it follows from $(\equiv)$ that both $\mathcal{M}_{A} \not \models p_{1} \equiv p_{1} \vee\left(p_{1} \wedge p_{2}\right)$ and $\mathcal{M}_{A} \not \models p_{1} \equiv p_{1} \wedge\left(p_{1} \vee p_{2}\right)$. Since $\mathcal{M}_{A} \in \mathcal{N}$, neither \#Abs1 nor \#Abs2 are $\mathcal{N}$-valid. In order to add texture to the present case, we may take $p_{1}$ to be 'It is raining' and $p_{2}$ to be 'It is windy', where $\{a\}$ is a rainy weather state and $\{c\}$ is a windy weather state. It follows by the inclusive semantics that although $\{a, c\}$ exactly verifies 'It is raining and windy', and so $\{a, c\}$ also exactly verifies 'It is raining or both raining and windy', the same cannot be said for 'It is raining', thereby indicating a discrepancy between the set of exact verifiers for 'It is raining' and 'It is raining or both raining and windy'. Similar considerations show that the sentences 'It is raining' and 'It is raining and either raining or windy' have different exact verifiers, and so do not express the same proposition.

By invalidating \#Necs, \#Imps, \#Abs1, and \#Abs2, the inclusive state semantics satisfies the initial aim set out in Section 2 to provide a theory of propositional identity which respects differences in subject-matter. Given that the definition of $\mathcal{N}$-logical consequence is perfectly general, it is straightforward to compute the $\mathcal{N}$ validity of any of the propositional identity sentences in $\mathcal{L}$. We may then ask whether $\mathcal{N}$-validity yields an extensionally adequate theory of propositional identity given the aim to track subject-matter, while preserving as many of the Boolean identities as possible. Instead of attempting to provide a determinate answer for all identity 
sentences in $i d(\mathbb{L})$, the following section will begin by presenting considerations in favour of excluding \#Dist1 and \#Dist2 from the logic of propositional identity.

\section{Distribution Laws}

In addition to the counterexamples to \#Abs1 and \#Abs2 given above, the semantics admits counterexamples to \#Dist1 and \#Dist2. Letting $\mathcal{M}_{D}=\left\langle S_{D}, \subseteq,|\cdot|{ }_{D}\right\rangle$ with $S_{D}=\mathcal{P}(\{a, b, c, d, e, f\})$ where $\left|p_{1}\right|_{D}=\langle\{\{a\}\},\{\{b\}\}\rangle,\left|p_{2}\right|_{D}=\langle\{\{c\}\},\{\{d\}\}\rangle$, and $\left|p_{3}\right|_{D}=\langle\{\{e\}\},\{\{f\}\}\rangle$, for pairwise distinct $a, b, c, d, e$, and $f$, we may derive:

$$
\begin{aligned}
\left|p_{1} \vee\left(p_{2} \wedge p_{3}\right)\right|_{D}= & \langle\{\{a\},\{c, e\},\{a, c, e\}\},\{\{b, d\},\{b, f\},\{b, d, f\}\}\rangle ; \\
\left|\left(p_{1} \vee p_{2}\right) \wedge\left(p_{1} \vee p_{3}\right)\right|_{D}= & \frac{\langle\{\{a\},\{a, c\},\{a, e\},\{c, e\},\{a, c, e\}\},}{\{\{b, d\},\{b, f\},\{b, d, f\}\}\rangle ;} \\
\left|p_{1} \wedge\left(p_{2} \vee p_{3}\right)\right|_{D}= & \langle\{\{a, c\},\{a, e\},\{a, c, e\}\},\{\{b\},\{d, f\},\{b, d, f\}\}\rangle ; \\
\left|\left(p_{1} \wedge p_{2}\right) \vee\left(p_{1} \wedge p_{3}\right)\right|_{D}= & \langle\{\{a, c\},\{a, e\},\{a, c, e\}\}, \\
& \underline{\{\{b\},\{b, d\},\{b, f\},\{d, f\},\{b, d, f\}\}\rangle .}
\end{aligned}
$$

Given that the underlined sets of exact verifiers (falsifiers) are not identical, we may conclude by $(\equiv)$ that both $\mathcal{M}_{D} \not \forall A \vee(B \wedge C) \equiv(A \vee B) \wedge(A \vee C)$ and $\mathcal{M}_{D} \not=A \wedge(B \vee C) \equiv(A \wedge B) \vee(A \wedge C)$, and so neither \#Dist1 nor \#Dist2 are $\mathcal{N}$-valid. ${ }^{20}$ However, the intuitive basis for claiming that \#Dist1 and \#Dist2 do not respect sameness of subject-matter is not nearly as obvious as it is for the absorption laws. Since \#Dist1 and \#Dist2 respect sameness in modal profile, I will take there to be a presumption in favour of accepting \#Dist1 and \#Dist 2 on grounds of parsimony, drawing fewer distinctions in the absence of countervailing considerations. Nevertheless, there are powerful abductive reasons for taking counterexamples such as $\mathcal{M}_{D}$ seriously, excluding \#Dist1 and \#Dist2 from the logic of propositional identity rather than restricting the space of models so as to maintain their validity.

We may begin by observing that, modulo simplifying assumptions, the model above commands a degree of intuitive appeal. For instance, let $\{a\}$ be the state of the ball being black, $\{c\}$ be the state of the ball being round, and $\{e\}$ be the state of the ball being iron, where $p_{1}$ is 'The ball is coloured', $p_{2}$ is 'The ball is shaped', and $p_{3}$ is 'The ball is metallic'. It follows that the fusion state $\{a, c\}$ of the ball being black and round fails to exactly verify 'The ball is coloured or both shaped and metallic'. This conclusion follows from the observations that: (1) $\{a, c\}$ includes $\{c\}$ as a part, and so does not exactly verify 'The ball is coloured' since $\{c\}$ is irrelevant; (2) $\{a, c\}$ is not a fusion of exact verifiers for 'The ball is shaped' and 'The ball is metallic', and so does not exactly verify 'The ball is shaped and metallic'; and (3) $\{a, c\}$ is not a fusion of exact verifiers for 'The ball is coloured' and 'The ball is shaped and

\footnotetext{
${ }^{20}$ Correia [9, p. 111-2] presents an analogous counterexample to \#Dist1 articulated in terms of his supersentence semantics, but does not extend similar considerations to \#Dist2 as above, claiming instead that \#Dist2 is valid. See Section 5 for further discussion.
} 
metallic', and so does not exactly verify 'The ball is coloured and both shaped and metallic'. Without changing the inclusive semantics, $\{a, c\}$ does not exactly verify 'The ball is coloured or both shaped and metallic'. Nevertheless, it is easy to see that $\{a, c\}$ exactly verifies 'The ball is coloured or shaped' on account of exactly verifying 'The ball is coloured and shaped', and so $\{a, c\}$ exactly verifies 'The ball is both coloured or shaped, and coloured or metallic'. Thus we may conclude that $\mathcal{M}_{D}$ provides an intuitively compelling counterexample to \#Dist1, where something similar may be shown for \#Dist2, making it unnatural to exclude these cases.

Insofar as validity over the class of normal models may claim to be reasonably natural, arbitrary models of $\mathcal{L}$ should not be excluded from consideration by fiat alone. Rather than attempting to hand pick the models of $\mathcal{L}$ which are to be considered in evaluating the validity of identity sentences, one may attempt to provide motivation for adopting a principled restriction on the class of models. In particular, Fine [13, 14] considers imposing the following restriction on models of the language:

Regular Contents: $\mathbb{C}_{\mathcal{S}}^{\mathrm{R}}=\left\{X \in \mathbb{C}_{\mathcal{S}}: y \in X\right.$ if $x \sqsubseteq y \sqsubseteq z$ for some $\left.x, z \in X\right\}$. Regular Propositions: $\mathbb{P}_{\mathcal{S}}^{\mathrm{R}}=\left\{\langle X, Y\rangle: X, Y \in \mathbb{C}_{\mathcal{S}}^{\mathrm{R}}\right\}$.

Regularity: An $\mathcal{S}$-model $\mathcal{M}$ is regular iff $\mathcal{M} \in \mathcal{N}$ and $|p| \in \mathbb{P}_{\mathcal{S}}^{\mathrm{R}}$ for all $p \in \mathbb{L}$.

In order to provide motivation for restricting consideration to regular models, Fine [14] appeals to the simplicity of the space of regular propositions, writing:

Regular propositions have an especially simple form. For each such proposition $P$ (if non-empty) will have a maximal verifier $p$, the fusion of all its verifiers, which we identify with its subject-matter - the agglomeration of the facts, so to speak, from which its verifiers are drawn; and it will also have various low lying verifiers, with all other verifiers lying above them. The proposition itself will then consist of all the states that lie between the low lying verifiers and the maximal verifier. Regular propositions are therefore subject to a limited form of monotonicity; given that a state verifies a regular proposition then so does any extension of the state as long as it lies within the subjectmatter of the proposition.

(p. 628-9)

It is worth considering the manner in which the limited form of monotonicity to which Fine refers fails to hold in the counterexamples to \#Dist1 and \#Dist2. In particular, with respect to \#Dist1, the outlier states in $\left|\left(p_{1} \vee p_{2}\right) \wedge\left(p_{1} \vee p_{3}\right)\right|_{D}^{+}$ all lie between states in $\left|p_{1} \vee\left(p_{2} \wedge p_{3}\right)\right|_{D}^{+}$, despite failing to be members of $\left|p_{1} \vee\left(p_{2} \wedge p_{3}\right)\right|_{D}^{+}$, and so $\left|p_{1} \vee\left(p_{2} \wedge p_{3}\right)\right|_{D}$ fails to be regular. Of course, something similar may be said for \#Dist 2 since $\left|p_{1} \wedge\left(p_{2} \vee p_{3}\right)\right|_{D}$ also fails to be regular.

Despite the fact that neither $\left|p_{1} \vee\left(p_{2} \wedge p_{3}\right)\right|_{D}$ nor $\left|p_{1} \wedge\left(p_{2} \vee p_{3}\right)\right|_{D}$ are regular, we may observe that $\mathcal{M}_{D}$ is a regular model insofar as all of the sentences letters are assigned to regular propositions. Even so, the complex sentences $p_{1} \vee\left(p_{2} \wedge p_{3}\right)$ and $p_{1} \wedge\left(p_{2} \vee p_{3}\right)$ do not express regular propositions, and so the class of regular models $\mathcal{R}$ fails to be uniform over the inclusive semantics. Rather than giving up regularity, one may maintain uniformity by adopting the following alternatives:

$(\wedge)_{\mathrm{S}}^{-} \quad \mathcal{M}, s \dashv A \wedge B$ iff $t \sqsubseteq s \sqsubseteq d$ for some $t$ and $d$ where $\mathcal{M}, d \dashv A \vee B$ and either $\mathcal{M}, t \dashv A$ or $\mathcal{M}, t \dashv B$. 
$(\vee)_{\mathrm{S}}^{+} \mathcal{M}, s \Vdash A \vee B$ iff $t \sqsubseteq s \sqsubseteq d$ for some $t$ and $d$ where $\mathcal{M}, d \Vdash A \wedge B$ and either $\mathcal{M}, t \Vdash A$ or $\mathcal{M}, t \Vdash B$.

I will refer to the result of replacing $(\wedge)^{-}$and $(\vee)^{+}$in the inclusive semantics with the clauses above as the super inclusive semantics. We may show that $\mathcal{R}$ is uniform over the super inclusive semantics so that for any $\mathcal{S}$-model $\mathcal{M} \in \mathcal{R}$ and $A \in \operatorname{ext}(\mathbb{L})$, the proposition $|A| \in \mathbb{P}_{\mathcal{S}}^{\mathrm{R}}$. Additionally, \#Dist1 and \#Dist 2 are $\mathcal{R}$-valid over the super inclusive semantics, though these validities come at the cost of a significant increase in the complexity of the semantics for conjunction and disjunction. Even supposing that the space of regular propositions could be shown to be simpler than the space of normal propositions, we must nevertheless weigh this increase in simplicity against the increase in complexity of the super inclusive semantics.

Given the super inclusive semantics together with a regular model $\mathcal{M} \in \mathcal{R}$, we may refer to the elements of $|A|^{+}$as the liberal verifiers for $A$ in $\mathcal{M}$, and refer to the elements of $|A|^{-}$as the liberal falsifiers for $A$ in $\mathcal{M} .^{21}$ In addition to the increase in overall complexity, it is natural to object that the super inclusive semantics does not provide the most compelling falsity-condition for conjunction, nor truth-condition for disjunction. For instance, in the example above, both $\{a\}$ and $\{c, e\}$ liberally verify 'The ball is coloured or both shaped and metallic', but the same cannot be said for $\{c\}$ considered on its own. However, given that $\{c\}$ is part of $\{a, c, e\}$, it follows that $\{a, c\}$ liberally verifies 'The ball is coloured or both shaped and metallic'. By contrast, $\{a, c\}$ does not exactly verify 'The ball is coloured or both shaped and metallic' on account of including $\{c\}$ as a part which makes no contribution to the truth of the sentence when considered on its own, or even when fused with $\{a\}$. Whereas $\{a, c, e\}$ overdetermines the truth of 'The ball is coloured or both shaped and metallic' on account of being the fusion of more than one state which makes the sentence true, the same cannot be said for $\{a, c\}$ since it is only when fused with $\{e\}$ that $\{c\}$ may be said to contribute to making 'The ball is coloured or both shaped and metallic' true. Accordingly, without being fused to $\{e\}$, the state $\{c\}$ is irrelevant to the truth of 'The ball is coloured or both shaped and metallic', and so $\{c\}$ without $\{e\}$ disqualifies $\{a, c\}$ as an exact verifier for the sentence in question.

Even if one were to relax the notion of relevance holding between states and the sentences which they make true or false, the super inclusive semantics faces a further difficulty. In particular, one must restrict attention to the class $\mathcal{R}^{+}$of nonvacuous regular models of $\mathcal{L}$ which may be defined as:

Nonvacuous: An $\mathcal{S}$-model $\mathcal{M}$ is nonvacuous iff both $|p|^{ \pm} \neq \varnothing$ for all $p \in \mathbb{L}$.

Without restricting consideration to just the nonvacuous regular models, we may let $\mathcal{M}_{E}=\left\langle S_{E}, \subseteq,|\cdot|_{E}\right\rangle$ with $S_{E}=\mathcal{P}(\{a, b, c\})$ for distinct elements $a, b$, and $c$, setting

\footnotetext{
${ }^{21}$ Although liberal verifiers (falsifiers) may be defined as the convex closure of the exact verifiers (falsifiers) for a sentence, exact verification (falsification) cannot be defined in terms of liberal verification (falsification) since different sets may have the same convex closure.
} 


$$
\begin{aligned}
\left|p_{1}\right|_{E}=\langle\varnothing,\{\{a\}\}\rangle, & \left|p_{2}\right|_{E}=\langle\{\{a\}\}, \varnothing\rangle, \text { and }\left|p_{3}\right|_{E}=\langle\{\{b\}\},\{\{c\}\}\rangle, \text { where: } \\
& \left|p_{1} \wedge p_{3}\right|_{E}=\langle\varnothing,\{\{a\},\{c\},\{a, c\}\}\rangle ; \\
& \left|p_{1} \vee p_{3}\right|_{E}=\langle\varnothing,\{\{a, c\}\}\rangle ; \\
& \left|p_{2} \vee p_{3}\right|_{E}=\langle\{\{a\},\{b\},\{a, b\}\}, \varnothing\rangle ; \\
& \left|p_{2} \wedge p_{3}\right|_{E}=\langle\{\{a, b\}\}, \varnothing\rangle .
\end{aligned}
$$

Since $p_{1}$ has no liberal verifiers, there are no liberal verifiers for $p_{1} \wedge p_{3}$, and so no state lies between a liberal verifier for $p_{1} \wedge p_{3}$ and a liberal verifier for either $p_{1}$ or $p_{3}$. Even though $\{b\}$ liberally verifies $p_{3}$, it follows by the super inclusive semantics for disjunction that there are no liberal verifiers for $p_{1} \vee p_{3}$, where a similar line of reasoning explains why there are no liberal falsifiers for $p_{2} \wedge p_{3}$ despite the fact that $\{c\}$ liberally falsifies $p_{3}$. But this is far from natural. By contrast, the inclusive semantics for disjunction maintains that any exact verifier for a disjunct ought to immediately qualify as an exact verifier for the disjunction to which it belongs, where similarly, the inclusive semantics for conjunction takes every exact falsifier for a conjunct to immediately qualify as an exact falsifier for the conjunction to which it belongs. Letting a proposition be vacuous just in case it either has no exact verifiers or no exact falsifiers, we may refer to the unnatural effects induced by the super inclusive semantics as vacuous annihilation. Although a proponent of the super inclusive semantics could prevent vacuous annihilation from occurring by restricting consideration to the class of nonvacuous regular models $\mathcal{R}^{+}$, there is nothing to motivate this restriction aside from the ambition to avoid vacuous annihilation. ${ }^{22}$

In addition to being ad hoc, the restriction to nonvacuous models trades on the assumption that the sentence letters in $\mathbb{L}$ are to be interpreted over the space of nonvacuous propositions. However, in opposition to this assumption, we may show that the restriction to nonvacuous propositions prevents the space of propositions from assuming an otherwise much more natural form. To begin with, one may expect that any theory of propositions ought to be bounded insofar as for any set of propositions $U$, there is a proposition $B$ which entails every proposition in $U$, and a proposition $T$ which is entailed by every proposition in $U$. By letting $U$ be the set of all propositions, we are guaranteed the existence of an upper bound which is entailed by all propositions, as well as a lower bound which entails all propositions. For instance, in an extensional theory of propositions, the upper and lower bounds on the space of propositions are the only propositions - namely, truth and falsity - where we may model these by $\{1\}$ and $\varnothing$, with subset inclusion for entailment. Similarly, intensional theories of propositions may be modelled by $\mathcal{P}(W)$ for any nonempty set $W$, where subset inclusion is entailment, and the sets $W$ and $\varnothing$ are the upper and lower bounds for $\mathcal{P}(W)$, respectively. Not only are such extensional and intensional theories of propositions bounded, this fact follows from their completeness, whereby any set $U$ of propositions has a least upper bound as well as a greatest lower bound with respect to entailment. Although completeness and boundedness could be given up,

\footnotetext{
${ }^{22}$ For instance, Fine [14, p. 649] considers restricting attention to nonvacuous propositions, providing a number of results which turn on this assumption.
} 
there is nothing to recommend making either of these further departures from tradition given the present ambition to provide a theory of propositions which respects differences in subject-matter. Thus in accordance with a conservative methodology, I will take there to be a strong presumption in favour of maintaining the boundedness and completeness of the present theory of propositions.

By contrast with extensional and intensional theories of propositions which are ordered by a single entailment relation, the present aim to accommodate differences in subject-matter yields a theory of propositions which admits of two distinct orders. To see where these orders come from, consider the abbreviated proofs from Boolean logics that conjunctive-parthood $A \sqsubseteq B:=A \wedge B \equiv B$ and disjunctive-parthood $A \leqslant B:=A \vee B \equiv B$ are each other's converse:

$$
\begin{aligned}
A \sqsubseteq B & \Rightarrow A \wedge B \equiv B \\
& \Rightarrow A \vee(A \wedge B) \equiv A \vee B \\
& \Rightarrow A \equiv A \vee B \\
& \Rightarrow B \vee A \equiv A \\
& \Rightarrow B \leqslant A . \\
A \leqslant B & \Rightarrow A \vee B \equiv B \\
& \Rightarrow A \wedge(A \vee B) \equiv A \wedge B \\
& \Rightarrow A \equiv A \wedge B \\
& \Rightarrow B \wedge A \equiv A \\
& \Rightarrow B \sqsubseteq A .
\end{aligned}
$$

Giving up \#Abs1 and \#Abs2 blocks the derivations above. Rather, $A \sqsubseteq B$ may hold without $B \leqslant A$ holding, and vice versa. For instance, although $A \sqsubseteq A \wedge B$ is valid, $A \wedge B \leqslant A$ may fail to hold, since $(A \wedge B) \vee A$ need not be wholly relevant to $A$, and so $(A \wedge B) \vee A \not \equiv A$. Similarly, although $A \leqslant A \vee B$ is valid, $A \vee B \sqsubseteq A$ need not hold, since $(A \vee B) \wedge A$ may fail to be wholly relevant to $A$, and so $(A \vee B) \wedge A \not \equiv A$. Whereas conjunctive-parthood and disjunctive-parthood are two ways of specifying the same order in a Boolean theory of propositions, these relations may come apart in the present setting. ${ }^{23}$ Nevertheless, a conservative methodology recommends that the space of propositions ought to be bounded above and below with respect to both orders $\sqsubseteq$ and $\leqslant$, where this feature follows from the stronger requirement that both orders over the space of propositions form complete lattices.

Insofar as disjunctive-parthood and conjunctive-parthood are taken to be complete lattices, we may show that both spaces of normal propositions $\mathbb{P}_{\mathcal{S}}$ and regular propositions $\mathbb{P}_{\mathcal{S}}^{\mathrm{R}}$ form bilattices, providing hyperintensional analogues of the Boolean lattices familiar from extensional and intensional logics. We may bring this out by defining semantic correlates of conjunctive-parthood, disjunctive-parthood, and negation as follows, where $X=\left\langle X^{+}, X^{-}\right\rangle$and $Y=\left\langle Y^{+}, Y^{-}\right\rangle$are propositions:

\footnotetext{
${ }^{23}$ As I argue elsewhere, ' $\leqslant$ ' and ' $\subseteq$ ' provide natural regimentations of constitutive explanatory readings of 'sufficient for' and 'necessary for'. See also [12] and [10].
} 
Essence: $X \sqsubseteq Y$ iff: (1) for every $b \in Y^{+}$there is some $a \in X^{+}$where $a \sqsubseteq b$;

(2) $a . b \in Y^{+}$whenever $a \in X^{+}$and $b \in Y^{+}$; and

(3) $X^{-} \subseteq Y^{-}$.

Ground: $X \leqslant Y$ iff: (1) for every $b \in Y^{-}$there is some $a \in X^{-}$where $a \sqsubseteq b$;

(2) $a . b \in Y^{-}$whenever $a \in X^{-}$and $b \in Y^{+}$; and

(3) $X^{+} \subseteq Y^{+}$.

Inversion: $\neg\left\langle X^{+}, X^{-}\right\rangle=\left\langle X^{-}, X^{+}\right\rangle$.

Given the definitions above, we may show for any normal model $\mathcal{M} \in \mathcal{N}$ that: (1) $\mathcal{M} \models A \sqsubseteq B$ just in case $|A| \sqsubseteq|B| ;$ (2) $\mathcal{M} \models A \leqslant B$ just in case $|A| \leqslant|B|$; and (3) $|\neg A|=\neg|A|$. Since $\mathcal{R} \subseteq \mathcal{N}$, these results also apply to the class of regular models. We may then consider the following definition:

Bilattice: A structure $\mathcal{B}=\langle\mathbb{P}, \sqsubseteq, \leqslant, \neg\rangle$ is a bilattice iff $\langle\mathbb{P}, \leqslant\rangle$ and $\langle\mathbb{P}, \sqsubseteq\rangle$ are complete lattices, $\mathbb{P}$ contains at least two elements, and that $\neg$ is a unary operator which satisfies all of the conditions: (1) $\neg \neg X=X$; (2) $X \leqslant Y=\neg X \sqsubseteq \neg Y$; and (3) $X \sqsubseteq Y=\neg X \leqslant \neg Y$.

The definition above was originally presented by Ginsberg [25, 26], and studied extensively by Fitting [19-24], among others. Whereas both $\mathcal{B}_{\mathcal{S}}=\left\langle\mathbb{P}_{\mathcal{S}}, \sqsubseteq, \leqslant, \neg\right\rangle$ and $\mathcal{B}_{\mathcal{S}}^{\mathrm{R}}=\left\langle\mathbb{P}_{\mathcal{S}}^{\mathrm{R}}, \sqsubseteq, \leqslant, \neg\right\rangle$ may be shown to be bilattices, we may nevertheless observe that the same cannot be said of the corresponding spaces of nonvacuous propositions, thereby falling short of what otherwise belongs to a natural class of well-studied mathematical structures. ${ }^{24}$

Even if one were to give up boundedness, and hence completeness - taking the space of propositions to form an unbounded bilattice in the sense studied by Bor and Rivieccio [5] — it is natural to maintain that each order forms a lattice over the space of propositions so that for any two propositions there is guaranteed to be both a least upper bound as well as a greatest lower bound. ${ }^{25}$ However, spaces of nonvacuous propositions do not form bilattices (unbounded or otherwise) since for any two propositions $X$ and $Y$ where no state is a part of either the exact verifiers or falsifiers for both $X$ and $Y$, there will fail to be a lower bound for $X$ and $Y$ with respect to either order, and so no greatest lower bound for $X$ and $Y$. Without admitting vacuous propositions, the space may at most be said to consist of two join-semilattices with at least two elements and a unary operator which satisfies the conditions (1) - (3) in the definition of a bilattice, though there is little to suggest that such structures constitute a reasonably natural class. ${ }^{26}$ Fine [14] makes a related observation, writing:

\footnotetext{
${ }^{24}$ This is not to claim that philosophers ought to be bound to what mathematicians have found to be most natural. Rather, I take it that without powerful motivation to do otherwise, a conservative methodology recommends beginning by thoroughly investigating appropriate applications of the most natural mathematical resources that have already been developed.

${ }^{25}$ An unbounded bilattice consists of two lattices with at least two elements together with a unary operator satisfying the conditions (1) - (3) in Bilattice above.

${ }^{26}$ Fine [14, p. 642] takes conjunction to be the greatest lower bound with respect to containment, flipping the perspective on conjunctive-parthood which I will maintain. See Section 6 for Fine's definition of containment along with a comparison to Essence.
} 
The domain of propositions has the structure of a lattice from a classical point of view and the structure (or something like the structure) of a bilattice from the present point of view.

Instead of following Fine (p. 649) in focusing on nonvacuous models and establishing a range of results which turn on nonvacuity, I will restrict attention throughout what follows to the normal and regular bilattices $\mathcal{B}_{\mathcal{S}}$ and $\mathcal{B}_{\mathcal{S}}^{\mathrm{R}}$.

Given the arguments above, a proponent of a regular theory of propositions cannot adopt either an inclusive or super inclusive semantics, at least insofar as the uniformity of $\mathcal{R}$ is to be maintained while avoiding nonvacuous collapse. In order to identify a suitable semantics for a regular theory of propositions, it will help to define the propositional operators which are expressed by ' $\wedge$ ' and ' $\vee$ ' when interpreted over the inclusive semantics, where $X, Y \in \mathbb{P}_{\mathcal{S}}$ are arbitrary normal propositions:

Content Fusion: $J \sqcap K=\{x . y: x \in J, y \in K\}$.

Conjunction: $X \wedge Y=\left\langle X^{+} \sqcap Y^{+}, X^{-} \cup Y^{-} \cup\left(X^{-} \sqcap Y^{-}\right)\right\rangle$.

Disjunction: $X \vee Y=\left\langle X^{+} \cup Y^{+} \cup\left(X^{+} \sqcap Y^{+}\right), X^{-} \sqcap Y^{-}\right\rangle$.

Given the inclusive semantics, we may show that for any normal model $\mathcal{M} \in \mathcal{N}$, both: (I) $|A \wedge B|=|A| \wedge|B|$; and (II) $|A \vee B|=|A| \vee|B|$. Moreover, we may show that $X \wedge Y$ and $X \vee Y$ are the least upper bounds of $X, Y \in \mathbb{P}_{\mathcal{S}}$ with respect to $\sqsubseteq$ and $\leqslant$, specifying clear theoretical roles for the semantics analogues of conjunction and disjunction to play within any bilattice of propositions $\mathcal{B}_{\mathcal{S}} \cdot{ }^{27}$

Although $\mathbb{P}_{\mathcal{S}}$ is closed under the operators $\wedge$ and $\vee$, the same cannot be said of $\mathbb{P}_{\mathcal{S}}^{R}$, where it is this fact together with (I) and (II) which explains why $\mathcal{R}$ fails to be uniform over the inclusive semantics. In order to maintain a regular theory of propositions, Fine [14, p. 632] considers the convex closure of the exact verifiers and falsifiers specified by the inclusive semantics, where the resulting semantic operations may be shown to be equivalent to the following:

Span: $[J, K]=\{y: x \sqsubseteq y \sqsubseteq z$ for some $x \in J$ and $z \in K\}$.

Convex Conjunction: $X \otimes Y=\left\langle X^{+} \sqcap Y^{+},\left[X^{-} \cup Y^{-},\left\{\bigsqcup\left(X^{-} \cup Y^{-}\right)\right\}\right]\right\rangle$.

Convex Disjunction: $X \otimes Y=\left\langle\left[X^{+} \cup Y^{+},\left\{\bigsqcup\left(X^{+} \cup Y^{+}\right)\right\}\right], X^{-} \sqcap Y^{-}\right\rangle$.

We may show that $\mathbb{P}_{\mathcal{S}}^{\mathrm{R}}$ is closed under $\otimes$ and $\oslash$, where $X \otimes Y$ and $X \otimes Y$ are the least upper bounds with respect to $\sqsubseteq$ and $\leqslant$ respectively, so long as $X$ and $Y$ are restricted to $\mathbb{P}_{\mathcal{S}}^{R}$. In place of the inclusive semantics, I will refer to the result of disjoining the super inclusive semantic clauses and the inclusive semantic clauses as the extremely inclusive semantics. Given any regular model $\mathcal{M} \in \mathcal{R}$, we may then show that $|A \wedge B|=|A| \otimes|B|$ and $|A \vee B|=|A| \otimes|B|$ hold with respect to the extremely inclusive semantics, making $\mathcal{R}$ uniform over the extremely inclusive semantics. Moreover, neither $\wedge$ nor $\vee$ result in vacuous annihilation given the extremely inclusive semantics, making the extremely inclusive semantics a superior alternative, at least insofar as a regular theory of propositions is to be maintained.

\footnotetext{
${ }^{27}$ These results may be taken to show that the semantic relations given in Essence and Ground are indeed the semantic correlates of conjunctive-part and disjunctive-part, respectively. One may also consider operators $\otimes$ and $\oplus$ for the greatest lower bounds with respect to $\sqsubseteq$ and $\leqslant$, referring to these as common essence and common ground, respectively.
} 
Recall Fine's claim from before that regular propositions have an especially simple form. As we have seen, the space of nonvacuous regular propositions does not satisfy the definition of a bilattice, and diverges from extensional and intensional theories of propositions in being unbounded and incomplete, where it is not clear what would motivate such departures. At the same time, admitting vacuous propositions makes $\mathcal{R}$ fail to be uniform over the inclusive semantics, motivating the super inclusive semantics which is uniform over $\mathcal{R}$. However, the super inclusive semantics gives rise to vacuous annihilation, providing a reason to adopt the much more complicated extremely inclusive semantics which avoids both of these defects. Nevertheless, the bilattice of regular propositions $\mathcal{B}_{\mathcal{S}}^{\mathrm{R}}$ satisfies the following conditions:

Interlaced: $\mathrm{A}$ bilattice $\mathcal{B}=\langle\mathbb{P}, \sqsubseteq, \leqslant, \neg\rangle$ is interlaced iff $(X \star Z) \circ(Y \star Z)$ if $X \circ Y$ where $\star \in\{\wedge \sqsubseteq, \leqslant, \vee \sqsubseteq, \vee \leqslant\}$ and $\circ \in\{\leqslant, \sqsubseteq\}$, where $\wedge^{\circ}$ and $\vee^{\circ}$ are the least upper bounds with respect to $\sqsubseteq$ and $\leqslant$.

Distributive: A bilattice $\mathcal{B}=\langle\mathbb{P}, \sqsubseteq, \leqslant, \neg\rangle$ is distributive iff whenever $\star, * \in\left\{\wedge^{\sqsubseteq}, \wedge^{\leqslant}, \vee^{\sqsubseteq}, \vee^{\leqslant}\right\}$, then $X \star(Y * Z)=(X \star Y) *(X \star Z)$.

By contrast with $\mathcal{B}_{\mathcal{S}}^{\mathrm{R}}$, the bilattice of normal propositions $\mathcal{B}_{\mathcal{S}}$ may fail to be interlaced, and so non-distributive since - as Fitting [21] observes - every distributive bilattice is interlaced. Although being distributive and interlaced are elegant properties for a bilattice to have, such virtues must be weighed against the increase in complexity of the extremely inclusive semantics. Even more importantly, we must ask which properties are appropriate given the application at hand. In addition to its added complexity, I take the counterexamples discussed at the beginning of the present section to show that the extremely inclusive semantics fails to provide natural semantic clauses for conjunction and disjunction for the same reasons given for the super inclusive semantics. In particular, one must weaken the manner in which states are required to be relevant to the sentences that they verifier or falsifier, adopting appropriate liberalisations of verification and falsification in place of the exact analogues. It is on these grounds that I will continue to maintain the inclusive semantics, extending consideration to all models in $\mathcal{N}$ as required by uniformity, and so will exclude \#Dist1 and \#Dist2 from the logic of propositional identity. Nevertheless, Interlaced and Distributive articulate at least one sense in which regular propositions may be said to enjoy a degree of simplicity that normal propositions do not.

Even in giving up \#Dist1 and \#Dist2 in addition to \#Abs1 and \#Abs2, on account of admitting counterexamples when evaluated over $\mathcal{N}$ given the inclusive semantics, the following principles may be shown to be $\mathcal{N}$-valid:
$\mathbf{A 1 0} A \wedge(A \vee B) \equiv A \vee(A \wedge B)$.
A11 $A \vee(B \wedge C) \leqslant(A \vee B) \wedge(A \vee C)$.
A12 $A \vee(B \wedge C) \sqsubseteq(A \vee B) \wedge(A \vee C)$.
A13 $A \wedge(B \vee C) \leqslant(A \wedge B) \vee(A \wedge C)$.
A14 $A \wedge(B \vee C) \sqsubseteq(A \wedge B) \vee(A \wedge C)$. 
Whereas A10 asserts that order does not matter in conjoining or disjoining a proposition $B$ with a proposition $A, \mathbf{A 1 1}$ - A14 provide analogues of \#Dist1 and \#Dist2 for disjunctive-parthood and conjunctive-parthood. Rather than offering an intuitive basis for accepting the principles above, I will take A10 - A14 to be justified by their $\mathcal{N}$-validity given the reasons presented above for adopting the inclusive semantics along with the full range of normal models included in $\mathcal{N}$. It remains to provide a broader description of the $\mathcal{N}$-logical consequence relation, surveying the space of $\mathcal{N}$-valid principles. In the following section, I will make a start by providing a logic for propositional identity which is sound over $\mathcal{N}$ given the inclusive semantics.

\section{A Logic for Propositional Identity}

Whereas Section 2 considered principles in which identity operators occurred within the scope of extensional operators, the syntax provided in Section 3 restricted attention to the propositional identity sentences in id $(\mathbb{L})$. Imposing this limitation simplified the semantics, while still providing a systematic means of evaluating identity sentences for validity. Given these syntactic constraints, I will refer to the proof system which results from combining the axioms A1 - A12 with the following rules of inference as The First-Degree Logic for Propositional Identity $\left(\mathrm{PI}^{1}\right)$ :

$$
\begin{array}{llll}
\text { R1 } & A \equiv B \vdash B \equiv A . & \text { R2 } & A \equiv B \vdash(A \wedge C) \equiv(B \wedge C) . \\
\text { R3 } & A \equiv B \vdash \neg A \equiv \neg B . & \text { R4 } & A \equiv B \vdash(A \vee C) \equiv(B \vee C) . \\
\text { R5 } & A \equiv B, B \equiv C \vdash A \equiv C . &
\end{array}
$$

I will take $\vdash_{\mathrm{PI}}^{1}$ to be the smallest relation to satisfy the axioms and rules of inference for $\mathrm{PI}^{1}$ which is closed under the standard structural rules, where $\varphi \in i d(\mathbb{L})$ is a theorem of $\mathrm{PI}^{1}$ just in case $\vdash_{\mathrm{PI}}^{1} \varphi$ as usual. It is straightforward to show that $\mathrm{PI}^{1}$ is sound with respect to the inclusive semantics and class $\mathcal{N}$ of normal models of $\mathcal{L}$. Accordingly, the motivation presented above for adopting the inclusive semantics and class of models $\mathcal{N}$ extends to each of the theorems of $\mathrm{PI}^{1}$. Additionally, R1 - R5 may be derived from Ref, Imps, and Func in a background classical propositional logic, providing further reason to accept these rules of inference as uncontroversial.

In addition to deriving A13 and A14 in $\mathrm{PI}^{1}$, we may show more generally that $\mathrm{PI}^{1}$ has the following duality property, where $\delta(\varphi)$ is the result of swapping all conjunction and disjunction signs in $\varphi$, and $\delta(\Gamma)=\{\delta(\gamma): \gamma \in \Gamma\}$ :

Duality: $\quad$ A logic $\Lambda$ is dual iff $\delta(\Gamma) \vdash_{\Lambda} \delta(\varphi)$ whenever $\Gamma \vdash_{\Lambda} \varphi$.

In contrast to $\mathrm{PI}^{1}$, Correia and Skiles [10] present a logic of generalised identity (GI), which is the result of both excluding $\mathbf{R 3}$ from $\mathrm{PI}^{1}$ while also including \#Dist2. Although Correia and Skiles explicitly exclude \#Dist1 from GI- thereby giving up duality - they do not offer any motivation for leaving \#Dist1 out when \#Dist2 has been included in GI. Instead, Correia and Skiles defer to the supersentence semantics provided by Correia [9] over which \#Dist2 may be shown to be valid, despite admitting counterexamples to \#Dist1. Although Correia says nothing to motivate the 
use of his semantics either by reference to a stock of principles which his semantics validates or else by means of an intended model, he nevertheless shows that his semantics is equivalent to a Finean inclusive state semantics for the extensional operators together with the following alternative clause for propositional identity:

$$
(\equiv)_{\mathrm{C}} \quad \mathcal{M} \models_{\mathrm{C}} A \equiv B \text { iff }|A|^{+}=|B|^{+} .
$$

Whereas ( $\equiv)$ required $A$ and $B$ to have the same exact verifiers and falsifiers in $\mathcal{M}$, Correia [9, p. 109] only requires $A$ and $B$ to have the same exact verifiers in $\mathcal{M}$, taking $\varphi \in \operatorname{id}(\mathbb{L})$ to be valid just in case $\mathcal{M} \models_{\mathrm{C}} \varphi$ for all $\mathcal{M} \in \mathcal{N}^{+}$- in symbols, $\models_{\mathrm{C}}^{\mathcal{N}^{+}} \varphi$ - where $\mathcal{N}^{+}$is the class of nonvacuous normal models of $\mathcal{L}{ }^{28}$ Correia [9] does not, however, provide any explicit motivation for adopting $(\equiv)_{\mathrm{C}}$ over the semantics given in $(\equiv)$, nor for restricting consideration to nonvacuous models, despite how much turns on these choices. Having already considered the demerits of restricting consideration to nonvacuous models, the present section will focus on the results of adopting Correia's semantics for propositional identity.

If $\mathbb{P}_{\mathcal{S}}$ is to model the space of propositions expressed by the sentences of a language where ' $\equiv$ ' expresses propositional identity, it is natural to assume that $A \equiv B$ is true in an $\mathcal{S}$-model just in case $A$ and $B$ are assigned to the same object inside $\mathbb{P}_{\mathcal{S}}$ by that model. Indeed, this is precisely what $(\equiv)$ asserts. By contrast, $(\equiv)^{+}$only requires two sentences to have the same exact verifiers for identity to hold, and so $A \equiv B$ may be true in a model for Correia despite the fact that $|A| \neq|B|$. This leads to a number of undesirable effects, perhaps most vividly exhibited by the invalidity of R3. In particular, counterexamples to $\mathbf{R} 3$ may be naturally extended to counterexamples to Func from which it follows that the negation operator is opaque, and so it follows by $\mathbf{P} 2$ that $\mathbf{L L}$ does not hold without exception. ${ }^{29}$ Despite these surprising results, Correia and Skiles say nothing to acknowledge or defend the conclusion that negation is an opaque operator. By contrast, I will take the extensional operators ' $\neg$ ', ' $\wedge$ ', and ' $\vee$ ' to be paradigm cases of transparent operators, maintaining a classical reading of ' $\rightarrow$ ' in Func and $\mathbf{L} \mathbf{L} .{ }^{30}$

The fact that Correia and Skiles cannot claim that Func and LL hold without exception in a language with operators for propositional identity and the extensional operators is a direct result of the asymmetry between truth and falsity which Correia [9] includes in his semantics. By contrast, it is easy to see why $\mathbf{R 3}$ comes out valid when one takes both the truth-conditions and falsity-conditions for sentences into consideration in evaluating propositional identity claims as in (三) from Section 3. Assuming $A \equiv B$ is true in an arbitrary model $\mathcal{M}$, it follows from $(\equiv)$ that $A$ and $B$ have the same exact verifiers and falsifiers. Given that negation

\footnotetext{
${ }^{28}$ Fine and Jago [18, p. 539] present a system of exact entailment where exact entailment is defined solely in terms of exact verification with corresponding implications for distribution, where $A \vee(B \wedge C)$ exactly entails $(A \vee B) \wedge(A \vee C)$ but not vice versa, while $A \wedge(B \vee C)$ and $(A \wedge B) \vee(A \wedge C)$ exactly entail each other, disrupting an otherwise natural duality.

${ }^{29}$ Similar problems arise for Correia's [8] semantics of analytic containment.

${ }^{30}$ Other transparent operators include the metaphysical modals ' $\square$ ' and ' $\diamond$ ', constitutive operators for essence and ground ' $\sqsubseteq$ ' and ' $\leqslant$ ', as well as the propositional identity operator ' $\equiv$ ' itself.
} 
inverts the exact verifiers and falsifiers, $\neg A$ and $\neg B$ will also have the same exact verifiers and falsifiers, and so $\neg A \equiv \neg B$ will come out true in $\mathcal{M}$. Of course, this argument breaks down if the truth of $A \equiv B$ only requires the sameness of the truth-conditions. As brought out by $\mathcal{M}_{D}$ above, two sentences may share the same truth-condition without sharing the same falsity-condition, and so the fact that $A$ and $B$ share the same truth-condition says nothing of whether $\neg A$ and $\neg B$ share the same truth-condition. Despite limiting consideration to truth-conditions in evaluating propositional identity claims, Correia maintains consideration of both truth-conditions and falsity-conditions in evaluating the extensional sentences in $\operatorname{ext}(\mathbb{L})$, making it all the more mysterious what motivates the sudden asymmetry late in his semantics. ${ }^{31}$ Rather than maintaining an asymmetry between the truthconditions and falsity-conditions in the semantics for propositional identity, I will take the validity of $\mathbf{R 3}$ to be an immediate consequence of $(\equiv)$ together with $(\neg)^{+}$ and $(\neg)^{-}$, from which it follows that negation is a transparent operator.

\section{Subject-Matter Revisited}

Recall from Section 2 the manner in which differences in subject-matter were taken to indicate differences between propositions. In particular, \#Necs, \#Imps, \#Abs1, and \#Abs2 were found to admit of exceptions, both motivating and constraining the development of the state semantics for $\mathcal{L}$ presented in Section 3. I then argued in Section 4 that there are strong abductive reasons for taking exception to \#Dist1 and \#Dist2, where Section 5 presented a logic of propositional identity which is sound over the semantics. It remains, however, to extend the language $\mathcal{L}$ to include the subject-matter operator ' $\sigma$ ', providing both a semantics and logic for ' $\sigma$ ', thereby supplying a more substantial theory of subject-matter than the collection of principles initially presented in Section 2.

We may begin by considering the extension $\mathcal{L}^{\sigma}$ of $\mathcal{L}$, defining the pre-identity sentences of $\mathcal{L}^{\sigma}$ as follows, where $p \in \mathbb{L}$ is arbitrary:

$$
A::=p|\neg A| \sigma A|A \wedge A| A \vee A \text {. }
$$

Letting pid $(\mathbb{L})$ be the set of pre-identity sentences of $\mathcal{L}^{\sigma}$, I will take $A \equiv B$ to be an identity sentence in $\mathcal{L}^{\sigma}$ for any $A, B \in \mathrm{pid}(\mathbb{L})$, where $\mathrm{id}^{\sigma}(\mathbb{L})$ is the set of all identity sentences in $\mathcal{L}^{\sigma}$, and eq $(\mathbb{L}) \subseteq i d^{\sigma}(\mathbb{L})$ is the set of all equivalences in $\mathcal{L}^{\sigma}$ of the form $A \leftrightarrow B$. In addition to maintaining the inclusive semantics defended in Section 3 together with the clause $(\equiv)$ for propositional identity, we may now consider the following clauses for the subject-matter operator:

$(\sigma)_{\mathrm{C}}^{+} \quad \mathcal{M}, s \Vdash \sigma A$ iff $x \sqsubseteq s \sqsubseteq y$ for some $x, y \in S$ where $\mathcal{M}, x \Vdash A$ and $\mathcal{M}, y \Vdash A$. $(\sigma)_{\mathrm{C}}^{-} \quad \mathcal{M}, s-\sigma A$ iff $x \sqsubseteq s \sqsubseteq y$ for some $x, y \in S$ where $\mathcal{M}, x-A$ and $\mathcal{M}, y-A$.

\footnotetext{
${ }^{31}$ Although one could define $A \equiv B:=\left(A \equiv_{\mathrm{C}} B\right) \wedge\left(\neg A \equiv_{\mathrm{C}} \neg B\right)$ in a less syntactically restricted language, or uniquely characterise $\equiv$ with the rules $A \equiv_{\mathrm{C}} B, \neg A \equiv_{\mathrm{C}} \neg B \vdash A \equiv B, A \equiv B \vdash A \equiv_{\mathrm{C}} B$, and $A \equiv B \vdash \neg A \equiv_{\mathrm{C}} \neg B$, one must ask why $\equiv_{\mathrm{C}}$ has be axiomatised rather than propositional identity $\equiv$. I am grateful to Kit Fine and Tim Williamson for bringing these points to my attention.
} 
A set of states $X$ is convex just in case $y \in X$ whenever $x \sqsubseteq y \sqsubseteq z$ for some $x, z \in X$. Letting $\lceil X\rceil=\{y: x, z \in X$ and $x \sqsubseteq y \sqsubseteq z\}$ be the convex closure of $X$, it is easy to show that $\left\lceil\lceil X\rceil=\lceil X\rceil .{ }^{32}\right.$ We may then refer to the semantic clauses given above as the convex semantics for $\sigma$ since the exact verifiers (falsifiers) for $\sigma A$ is the convex closure of the exact verifiers (falsifiers) for $A$. Letting $\mathrm{PI}_{\mathrm{C}}^{1}$ be the result of including the following axioms and rule of inference in $\mathrm{PI}^{1}$, we may show that given the convex semantics, $\mathrm{PI}_{\mathrm{C}}^{1}$ is sound over the class of nonvacuous normal models $\mathcal{N}^{+}$of $\mathcal{L}^{\sigma}$ :
R6 $A \equiv B \vdash A \leftrightarrow B$.
S9 $\quad A \vee(B \wedge C) \leftrightarrow(A \vee B) \wedge(A \vee C)$
S8 $\sigma \sigma A \equiv \sigma A$.
$\mathbf{S 1 0} A \wedge(B \vee C) \leftrightarrow(A \wedge B) \vee(A \wedge C)$.

It is worth noting that $\mathbf{R 6}$ makes ' $\sigma$ ' a transparent operator so that $\sigma$ operates solely on the propositions expressed by the sentences to which ' $\sigma$ ' is appended, thereby capturing Obj given in Section 2. Additionally, S3 - S7 stated in Section 2 follow immediately from $\mathbf{R 6}$ given the range of identities included in $\mathrm{PI}^{1}$.

Were one to extend consideration to all normal models in $\mathcal{N}$, both $\mathbf{S 9}$ and $\mathbf{S 1 0}$ will admit of counterexamples. For instance, letting $\mathcal{M}_{F}=\left\langle S_{F}, \subseteq, \mid \cdot{ }_{F}\right\rangle$ with $S_{F}=\mathcal{P}(\{a, b, c, d, e, f\})$ where $\left|p_{1}\right|_{F}=\langle\{\{a\}\},\{\{b\}\}\rangle,\left|p_{2}\right|_{F}=\langle\{\{c\}\},\{\{d\}\}\rangle$, $\left|p_{3}\right|_{F}=\langle\varnothing,\{\{e\}\}\rangle$, and $\left|p_{4}\right|_{F}=\langle\{\{f\}\}, \varnothing\rangle$, for pairwise distinct $a, b, c, d, e$, and $f$, we may derive the following identities:

$$
\begin{aligned}
& \left|p_{1} \vee\left(p_{2} \wedge p_{3}\right)\right|_{F}=\langle\{\{a\}\},\{\{b, d\},\{b, e\},\{b, d, e\}\}\rangle ; \\
& \left|\left(p_{1} \vee p_{2}\right) \wedge\left(p_{1} \vee p_{3}\right)\right|_{F}=\langle\{\{a\},\{c\},\{a, c\}\},\{\{b, d\},\{b, e\},\{b, d, e\}\}\rangle ; \\
& \left|p_{1} \wedge\left(p_{2} \vee p_{4}\right)\right|_{F}=\langle\{\{a, c\},\{a, f\},\{a, c, f\}\},\{\{b\}\}\rangle ; \\
& \left|\left(p_{1} \wedge p_{2}\right) \vee\left(p_{1} \wedge p_{4}\right)\right|_{F}=\langle\{\{a, c\},\{a, f\},\{a, c, f\}\},\{\{b\},\{d\},\{b, d\}\}\rangle .
\end{aligned}
$$

Since the convex closures of the underlined sets are not identical, neither $\mathbf{S 9}$ nor $\mathbf{S 1 0}$ is $\mathcal{N}$-valid on the convex semantics. Although one could exclude such counterexamples by restricting consideration to the nonvacuous models in $\mathcal{N}^{+}$, imposing such restrictions faces the same criticism brought out in Section 4. Accordingly, without justifying the restriction to $\mathcal{N}^{+}$, the convex semantics cannot provide a basis of support for the claim that \#Dist1 and \#Dist2 respect sameness of subject-matter.

Despite the shortcomings faced by the convex semantics, we may nevertheless observe that ' $\leftrightarrow$ ' has the same derived semantic clause as the semantics which Fine [13] provides for synonymy in the first degree fragment of Angell's [2] logic of analytic containment (AC), or what Fine calls analytic equivalence, where we may take $\lceil A\rceil^{ \pm}:=\left\lceil|A|^{ \pm}\right\rceil$to be the convex closure of $|A|^{ \pm}$for ease of exposition:

$$
(\leftrightarrow) \quad \mathcal{M} \models A \leftrightarrow B \text { iff }\lceil A\rceil^{ \pm}=\lceil B\rceil^{ \pm} .
$$

Whereas synonymy asserts the identity of the meanings of two terms - in this case sentences - the same cannot be said for sameness of subject-matter. In particular,

\footnotetext{
${ }^{32}$ Alternatively, one could define $\lceil X\rceil=[X, X]$, where $[X]$ is defined in Span from Section 3 above.
} 
both $\mathbf{S 1}$ and $\mathbf{S 2}$ reproduced below ought to come out valid:

S1 $\neg A \leftrightarrow A$. S2 $A \wedge B \leftrightarrow A \vee B$.

Although $\neg A$ and $A$ have the same subject-matter, they do not have the same meaning, where something similar may be said of $A \wedge B$ and $A \vee B$. However, neither S1 nor $\mathbf{S 2}$ is valid over the convex semantics. Not only do these considerations provide good reason to reject the convex semantics for subject-matter, they also raise doubts for Angell's [2] logic AC. Given that synonymy in AC is coextensive with sameness of subject-matter in $\mathrm{PI}_{\mathrm{C}}^{1}$, and that distinct propositions may nevertheless have the same subject-matter in $\mathrm{PI}_{\mathrm{C}}^{1}$, it follows that synonymy in $\mathrm{AC}$ is not as fine-grained as propositional identity in $\mathrm{PI}_{\mathrm{C}}^{1}$. In particular, we may observe that although $\mathbf{S 9}$ and $\mathbf{S 1 0}$ have been included in $\mathrm{PI}_{\mathrm{C}}^{1}$, neither \#Dist1 nor \#Dist2 belong to $\mathrm{PI}_{\mathrm{C}}^{1}$. However, this is far from natural, since one may expect synonymy to be at least as fine-grained as propositional identity, if not much more fine-grained on account of the same propositions being expressed by sentences with different meanings. ${ }^{33}$

Despite the disparity between the theoretical targets for analytic equivalence and propositional identity, it is worth reviewing Fine's reasons for taking the identity of the convex closures of the exact verifiers (falsifiers) for $A$ and $B$ to provide a semantics for analytic equivalence rather than the identity of sets of exact verifiers (falsifiers) for $A$ and $B$. To begin with, Fine defines containment:

Containment: $\quad T<U$ iff: (1) for all $u \in U$, there is some $t \in T$ where $t \sqsubseteq u$; and (2) for all $t \in T$, there is some $u \in U$ where $t \sqsubseteq u$.

Instead of providing an independent theoretical target for containment, Fine [13] takes containment to provide a semantics for a unilateral notion of analytic entailment which only concerns exact verifiers, writing:

Containment is the relation between contents which is the analogue of the relation of analytic entailment between statements. Thus we will want to say that $A$ analytically entails $C$ just in case the content of $A$ contains the content of $C$. [...] If the relation $T<U$ is genuinely to represent a relation of partial content, of $T$ being part of the content $U$, then we would expect the relation to be antisymmetric.

(p. 207-8)

Even in supposing there were a clear theoretical target by which to evaluate accounts of analytic entailment, Fine says nothing in support of the claim that containment is the most natural notion of partial content, or that it provides the most natural semantics for analytic entailment. Nevertheless, it is clear that Fine takes antisymmetry to be essential to any genuine parthood relation, and so given this assumption, antisymmetry is required of containment insofar as containment is to be a notion of partial content. Although Fine (p. 208) shows that containment is only antisymmetric for convex sets of states, he does not provide a reason to restrict attention to convex sets of states rather than revising his definition of containment.

\footnotetext{
${ }^{33}$ Fine [13] proves that $\mathrm{AC}$ is sound and complete over his semantics, and so $\varphi \in \mathrm{eq}(\mathbb{L})$ is a theorem of $\mathrm{PI}_{\mathrm{C}}^{1}$ just in case $\varphi$ is valid over $\mathcal{N}^{+}$given the convex semantics.
} 
In addition to these motivational concerns, we may note that Fine (p. 201) defines analytic entailment as $A \rightarrow B:=A \leftrightarrow A \wedge B$, where ' $\leftrightarrow$ ' expresses analytic equivalence. Although Fine takes ' $\leftrightarrow$ ' to be a primitive term instead of being defined in terms of ' $\sigma$ ' as above, the semantics that Fine (p. 217) provides for ' $\triangleleft$ ' is equivalent to $(\triangleleft)$ derived above. We may then follow Fine in deriving the following semantic clause from the definitions of analytic equivalence and containment for all $\mathcal{M} \in \mathcal{N}^{+}$:

$$
(\rightarrow) \quad \mathcal{M} \models A \rightarrow B \text { iff }\lceil B\rceil^{+}<\lceil A\rceil^{+} \text {and }\lceil B\rceil^{-} \subseteq\lceil A\rceil^{-} \cdot 34
$$

Given any $A$ which has exact verifiers, and any $B$ which does not, $A \wedge B \rightarrow A$ will not hold, contrary to Fine's (p. 201) expectations. However, following Fine (p. 205) in restricting to nonvacuous models faces the same objections brought out in Section 4 above. By contrast, one may take conjunctive-parthood to provide a notion of partial content where ${ }^{\ulcorner} A \sqsubseteq B^{\urcorner}$reads ' $A$ is analytically entailed by $B^{\urcorner}$, observing that $A \sqsubseteq A \wedge B$ and $B \sqsubseteq A \wedge B$ are valid even without restricting consideration to nonvacuous models. Although it remains to specify a theoretical target for partial content against which conjunctive-parthood may be evaluated for adequacy, we may derive the following results, including disjunctive-parthood for comparison:

$$
\begin{array}{ll}
(\sqsubseteq) & \mathcal{M} \models A \sqsubseteq B \text { iff }|A| \sqsubseteq|B| . \\
(\leqslant) & \mathcal{M} \models A \leqslant B \text { iff }|A| \leqslant|B| .{ }^{35}
\end{array}
$$

So long as A1, A3, and R5 all hold, it is easy to show that conjunctive-parthood is antisymmetric. ${ }^{36}$ Accordingly, taking conjunctive-parthood to play the role of partial content avoids the need to close the exact verifiers and falsifiers for the propositions in question under convexity. Without an argument that $(\rightarrow)$ is preferable to $(\sqsubseteq)$ as a semantics for analytic entailment, the assumption that partial content ought to be antisymmetric does not provide a reason to require propositions to be convex, for as we have seen, conjunctive-parthood is antisymmetric even without closing the exact verifiers and falsifiers under convexity.

Setting aside the connections between sameness of subject-matter in $\mathrm{PI}_{\mathrm{C}}^{-}$and analytic equivalence in $\mathrm{AC}$, there is good reason to consider the undirected semantics for ' $\sigma$ ' on account of validating $\mathbf{S 1}$ over all normal models in $\mathcal{N}$ :

$$
\begin{aligned}
& (\sigma)_{\mathrm{U}}^{+} \quad \mathcal{M}, s \Vdash \sigma A \text { iff } s \sqsubseteq t \text { for some } t \in S \text { where } \mathcal{M}, t \Vdash A \vee \neg A . \\
& (\sigma)_{\mathrm{U}}^{-} \quad \mathcal{M}, s-\sigma A \text { iff } s \sqsubseteq t \text { for some } t \in S \text { where } \mathcal{M}, t \dashv A \wedge \neg A .
\end{aligned}
$$

\footnotetext{
${ }^{34}$ Fine [13, p. 217] considers the wider space of nonvacuous models where sentence letters may be assigned to propositions whose contents are nonempty but not necessarily closed under fusion.

${ }^{35}$ It is worth observing that the semantics for disjunctive-parthood is the result of inverting the exact verifiers and falsifiers in the semantics for conjunctive-parthood, thereby validating $A \sqsubseteq B \vdash \neg A \leqslant \neg B$ and $A \leqslant B \vdash \neg A \sqsubseteq \neg B$. See [14, p. 661] for related results.

${ }^{36}$ Proof: If $A \sqsubseteq B$ and $B \sqsubseteq A$, then $A \wedge B \equiv B$ and $B \wedge A \equiv A$, and so $A \equiv A \wedge B$ by $\mathbf{A 3}$ and R5. Thus $A \equiv B$ again by $\mathbf{R 5}$, where the converse is immediate from $\mathbf{A 1}$.
} 
On the undirected semantics, $\sigma A$ is both exactly verified and falsified by the parts of the exact verifiers and falsifiers for $A$. It also follows that both of the following principles are valid, where $\mathbf{S 1 2}$ follows from $\mathbf{S 1}$ and $\mathbf{S 1 1}$ by $\mathbf{R 5}$ :
S11 $\sigma A \equiv \neg \sigma A$.
$\mathbf{S 1 2} \sigma \neg A \equiv \neg \sigma A$.

Rather than taking ' $\sigma$ ' to be a sentential operator, Fine $[13,15,17]$ identifies the subject-matter of a proposition with the fusion of all exact verifiers and falsifiers for that proposition. Accordingly, the subject-matter of $A$ is not a proposition at all for Fine, but rather a single state, and so neither S11 nor S12 can be interpreted on Fine's semantics for subject-matter. Despite the disparity in kind between Fine's objectual account of subject-matter and the propositional account assumed above, the undirected semantics validates the same equivalences in eq $(\mathbb{L})$ as Fine's account of subject-matter. ${ }^{37}$ In particular, $\mathbf{S 2}$ is invalid over the class of normal models $\mathcal{N}$, contrary to the expectations above. For instance, if $A$ has no exact verifiers, then neither will $A \wedge B$, though $A \vee B$ will retain all of the exact verifiers for $B$, where a similar discrepancy may occur if $B$ has exact falsifiers but $A$ does not. Thus the parts of the exact verifiers and falsifiers for $A \wedge B$ may diverge from the parts of the exact verifiers and falsifiers for $A \vee B$, resulting in counterexamples to $\mathbf{S 2}$.

Rather than restricting attention to the nonvacuous models of $\mathcal{L}$, or else excluding S2 from the logic for subject-matter, I will provide a semantics which validates all of the subject-matter principles defended above. It will help to begin with a propositional analogue of another idea that Fine [13, 15, 17] develops, focusing at first on the positive subject-matter operator ' $\sigma^{+}$', In contrast to Fine who takes $\sigma^{+} A$ to be the fusion of the exact verifiers for $A$, I will draw on the duality operator ' $\delta$ ', defined in Section 5 in order to provide the following dual semantics for the positive subject-matter operator ' $\sigma^{+}$, , where ${ }^{\top} \sigma^{+} A^{\top}$ reads ' It is partially the case that $A^{\urcorner}: 38$

$$
\begin{array}{ll}
\left(\sigma^{+}\right)_{\mathrm{D}}^{+} & \mathcal{M}, s \Vdash \sigma^{+} A \text { iff } s \sqsubseteq t \text { for some } t \in S \text { where } \mathcal{M}, t \Vdash A \vee \delta(A) . \\
\left(\sigma^{+}\right)_{\mathrm{D}}^{-} & \mathcal{M}, s-\sigma^{+} A \text { iff } s \sqsubseteq t \text { for some } t \in S \text { where } \mathcal{M}, t \dashv A \wedge \delta(A) .
\end{array}
$$

Were we to omit ' $\delta(A)$ ' from the clauses above, $\sigma^{+}(A \wedge B)$ and $\sigma^{+}(A \vee B)$ will fail to be identical in cases where $A$ has exact verifiers but $B$ does not. However, given the semantics for conjunction and disjunction, it is natural to expect that it being partially the case that $A \wedge B$ is the same as it being partially the case that $A \vee B$. A similar point holds for the negative subject-matter operator which we may define by $\sigma^{-} A:=\sigma^{+} \neg A$, where ${ }^{\top} \sigma^{-} A^{\urcorner}$reads 'It is partially not the case that $A^{\urcorner}$.

Given the reading of ' $\sigma^{+}$' together with the definition of ' $\sigma^{-}$', we may define the subject-matter operator by $\sigma A:=\sigma^{+} A \vee \sigma^{-} A$, thereby incorporating the central idea behind the undirected semantics into a dual semantics for ' $\sigma$ '. Thus ' $\sigma A^{\text {' }}$ reads

\footnotetext{
${ }^{37}$ See [13, p. 209] and [27, p. 718] for similar observations.

${ }^{38}$ I will assume an improper reading of 'partially' so that it being the case that $A$ entails that it is partially the case that $A$. See also [15, p. 699] for notions of partial aboutness.
} 
'It is partly the case that $A$ or it is partly not the case that $A^{\top}$, where we may then derive the following dual semantic clauses for ' $\sigma$ ':

$(\sigma)_{\mathrm{D}}^{+} \quad \mathcal{M}, s \Vdash \sigma A$ iff $s \sqsubseteq t$ for some $t \in S$ where $\mathcal{M}, t \Vdash A \vee \neg A \vee \delta(A) \vee \delta(\neg A)$.

$(\sigma)_{\mathrm{D}}^{-} \mathcal{M}, s \dashv \sigma A$ iff $s \sqsubseteq t$ for some $t \in S$ where $\mathcal{M}, t \dashv A \wedge \neg A \wedge \delta(A) \wedge \delta(\neg A)$.

That the dual semantics validates both $\mathbf{S 1}$ and $\mathbf{S 2}$ over $\mathcal{N}$ should not surprise, for the exact verifiers for $\sigma A$ include all parts of the exact verifiers for $A, \neg A$, and their duals, where the same may be said of the exact falsifiers. Additionally, the dual semantics validates the following distribution laws:

$$
\text { S13 } \sigma(A \vee B) \equiv(\sigma A \vee \sigma B) . \quad \text { S14 } \sigma(A \wedge B) \equiv(\sigma A \wedge \sigma B) .
$$

Instead of revising the syntax for $\mathcal{L}^{\sigma}$, we may continue to take ' $\sigma$ ' to be primitive for present purposes, excluding ' $\sigma^{+}$' and ' $\sigma^{-}$' from $\mathcal{L}$. Nevertheless, we may draw on the definition of subject-matter given above in order to justify the informal reading of ${ }^{\ulcorner} \sigma A^{\urcorner}$. Additionally, we may extend the proof theory to accommodate the subjectmatter operator by letting $\mathrm{PI}_{\sigma}^{1}$ include $\mathrm{PI}^{1}$ along with R6, S1, S2, S8, S11, and S13, where we may then derive S3-S7, S9, S10, S12, and S14. ${ }^{39}$

Having begun to survey the space of subject-matter principles, it remains to provide an account of relevance. Were one to take the relevance operator ' $\leq$ ' to be primitive, Rel could be captured by including the following rule of inference in $\mathrm{PI}_{\sigma}^{1}$, where $\mathbf{L 1} \mathbf{-} \mathbf{L} \mathbf{4}$ could be included as axioms:

R7 $A \leftrightarrow B, C \leq A \vdash C \leq B$.

A natural motivation for $\mathbf{R 7}$ conceives of relevance as a parthood relation for subjectmatter, so that $A \leq B$ asserts that the subject-matter of $A$ is part of the subject-matter of $B$. Thus, if $A$ and $B$ share the same subject-matter, where the subject-matter of $C$ is part of the subject-matter of $A$, then the subject-matter of $C$ is also part of the subjectmatter of $B$. This justification for $\mathbf{R 7}$ can be strengthened by defining relevance as $A \preceq B:=\sigma A \leqslant \sigma B$, or equivalently, as $A \preceq B:=\sigma A \sqsubseteq \sigma B{ }^{40}$ Informally, $A$ is relevant to $B$ just in case the subject-matter of $A$ is a disjunctive-part of the subject-matter of $B$, or equivalently, the subject-matter of $A$ is a conjunctive-part of the subject-matter of $B$. Regardless of which convention one adopts, $\mathbf{R 7}$ and $\mathbf{L 1}-$ L4 may be derived in $\mathrm{PI}_{\sigma}^{1}$, where Obj and Rel follow from R6 and R7 by classical reasoning, thereby providing the beginnings of a theory of relevance. ${ }^{41}$

\footnotetext{
${ }^{39} \mathrm{It}$ is worth noting that every $\varphi \in \mathrm{eq}(\mathbb{L})$ is self-dual insofar as $\varphi \vdash_{\mathrm{PI}_{\sigma}}^{1} \delta(\varphi)$.

${ }^{40} \mathrm{By}$ contrast, there are the directed notions of positive relevance $A \leq{ }^{+} B:=\sigma^{+} A \leqslant \sigma^{+} B$ and negative relevance $A \leq^{-} B:=\sigma^{-} A \leqslant \sigma^{-} B$ (or equivalently, $A \leq^{-} B:=\sigma^{+} A \sqsubseteq \sigma^{+} B$ ) which do not assimilate what is relevant to $A$ and what is relevant to $\neg A$. For instance, as brought out in footnote 16 , although the exact verifier states for a sentence must be wholly relevant to that sentence, those states may fail to be wholly relevant to its negation.

${ }^{41}$ Alternatively, one may define relevance by either of the equivalent conventions $A \leq B:=A \wedge B \leqslant B$ or $A \leq B:=A \vee B \sqsubseteq B$. Although $\mathbf{L 1}-\mathbf{L} 4$ are easily derived, $\mathbf{R 7}$ must be explicitly included in $\mathrm{PI}_{\sigma}^{1}$.
} 
It is worth noting that relevance is not an entailment relation. Although both $A$ and $B$ are relevant to $A \wedge B$ by $\mathbf{L} \mathbf{2}$ and $\mathbf{L 4}$, neither $A$ nor $B$ entails $A \wedge B$. Accordingly, the present account of relevance is not to be assimilated to the notions of relevant entailment, or analytic implication, as developed by the relevance logicians. ${ }^{42}$ Although one could attempt to draw on a theory of relevance in combination with an account of implication in order to provide an analysis of a relevant implication relation, we may observe that disjunctive-part and conjunctive-part already amount to forms of relevant implication. In particular, we may derive the following:

$$
\text { T1 } A \leqslant B \vdash A \leq B . \quad \text { T2 } A \sqsubseteq B \vdash A \preceq B .
$$

If $A \leqslant B$, then by definition $A \vee B \equiv B$, and so $B$ obtains in any possibility in which $A$ obtains. Thus $A \leqslant B$ is an implication relation from $A$ to $B$, where the former must be relevant to the latter by $\mathbf{T 1}$. By contrast, we may observe that if $A \sqsubseteq B$, then by definition $A \wedge B \equiv B$, and so $A$ obtains in every possibility in which $B$ obtains. Accordingly, $A \sqsubseteq B$ is an implication relation from $B$ to $A$, where the latter must be relevant to the former by $\mathbf{T} 2$. Given that both relevance and implication flow from left to right for disjunctive-part, but in opposite directions for conjunctivepart, it is disjunctive-part rather than conjunctive-part which makes for the most apt comparison with the entailment relations studied in relevance logics.

In contrast to the rough outline presented in Section 2, the axioms and rules included in $\mathrm{PI}_{\sigma}^{1}$ provide a much richer theory of both subject-matter and relevance. Nevertheless, it remains to establish completeness over the semantics defended above, if the semantics has a complete logic at all. Additionally, it is desirable to provide a semantics that does not restrict consideration to the sentences in id $^{\sigma}(\mathbb{L})$, interpreting sentences which contain any combination of sentential operators included in the language. ${ }^{43}$ Nevertheless, maintaining the restriction to id $\mathrm{d}^{\sigma}(\mathbb{L})$ provides a first step towards a logic of propositional identity with greater expressive power. In particular, I will take $\mathrm{PI}_{\sigma}^{1}$ to provide an elegant theory of propositional identity with a well motivated semantics which is able to individuate propositions according to their subject-matter rather than their modal profile alone, thereby satisfying the aims set out above.

\section{Appendix}

I will begin by considering propositional languages of the form $\mathcal{L}=\langle\mathbb{L}, \overrightarrow{\mathcal{Q}}\rangle$ where each $\mathcal{Q}_{i}^{n} \in \overrightarrow{\mathcal{Q}}$ is an $n$-ary sentential operator for some $n \in \mathbb{N}$, and $\overrightarrow{\mathcal{Q}}$ includes the extensional connectives ' $\neg$ ', ' $\wedge$ ', and ' $\vee$ ' along with the propositional identity operator ' $\equiv$ '. The well-formed sentences in wfs $(\mathbb{L})$ consist of the sentence letters in $\mathbb{L}$ along with any $\mathcal{Q}_{i}^{n}(\vec{O})$, where $\mathcal{Q}_{i}^{n} \in \overrightarrow{\mathcal{Q}}$ and $\vec{O}$ is a sequence of $n$ well-formed sentences. Assuming a background classical logic in which ${ }^{\ulcorner} A \rightarrow B^{\urcorner}$abbreviates

\footnotetext{
${ }^{42}$ For instance, see [1, 2, 34].

${ }^{43}$ I provide such a semantics in [6].
} 
$\left\ulcorner\neg A \vee B^{\urcorner}\right.$, we may consider the following:

$$
\begin{array}{llll}
\text { Ref } & A \equiv A . & \text { Trans } & (A \equiv B) \rightarrow[(B \equiv C) \rightarrow(A \equiv C)] . \\
\text { Sym } & (A \equiv B) \rightarrow(B \equiv A) . & \text { Imps } & (A \equiv B) \rightarrow(A \rightarrow B) . \\
\text { LL } & (A \equiv B) \rightarrow\left(C \rightarrow C_{(B / A)}\right) . & \text { Func } & (A \equiv B) \rightarrow\left[\mathcal{Q}(\vec{O}) \equiv \mathcal{Q}\left(\vec{O}_{(B / A)}\right)\right] .
\end{array}
$$

As above, $\mathcal{L}$ is transparent just in case all instances of Func hold, where ${ }^{r} \mathcal{Q}\left(\vec{O}_{(B / A)}\right)^{\urcorner}$ is the result of replacing one or more instances of ${ }^{\top} A^{\top}$ which occur as members of the sequence ${ }^{\ulcorner} \vec{O}^{\urcorner}$with ${ }^{\ulcorner} B^{\urcorner}$, where similarly ${ }^{\ulcorner} \mathcal{Q}\left(\vec{O}_{[B / A]}\right)^{\urcorner}$is the result of replacing all instances of ${ }^{\ulcorner} A^{\urcorner}$which occur as members of the sequence ${ }^{\ulcorner} \vec{O}^{\urcorner}$with ${ }^{\ulcorner} B^{\urcorner}$. Additionally, I will take ${ }^{\top} C_{(B / A)}{ }^{\top}$ to be the result of replacing one or more instances of ${ }^{\top} A^{\top}$ as it occurs anywhere in ${ }^{\ulcorner} C^{\urcorner}$with ${ }^{\ulcorner} B{ }^{\urcorner}$, as well as taking ${ }^{\ulcorner} C_{[B / A]}{ }^{\top}$ to be the result of replacing all instances of ${ }^{\ulcorner} A^{\urcorner}$as it occurs anywhere in ${ }^{\ulcorner} C^{\urcorner}$with ${ }^{\ulcorner} B^{\urcorner}$. We may now prove the following propositions.

P1 If $\mathcal{L}$ is transparent, then Ref and Imps entail both Sym and Trans.

Proof Assuming that $\mathcal{L}$ is transparent, $(A \equiv B) \rightarrow[(A \equiv A) \equiv(B \equiv A)]$ follows, where $[(A \equiv A) \equiv(B \equiv A)] \rightarrow[(A \equiv A) \rightarrow(B \equiv A)]$ holds by Imps, and so $(A \equiv B) \rightarrow(B \equiv A)$ follows from Ref by propositional logic. Again by transparency, $(A \equiv B) \rightarrow([(A \equiv C) \rightarrow(A \equiv C)] \equiv[(B \equiv C) \rightarrow(A \equiv C)])$, and so $(A \equiv B) \rightarrow([(A \equiv C) \rightarrow(A \equiv C)] \rightarrow[(B \equiv C) \rightarrow(A \equiv C)])$ by Imps. Since $(A \equiv C) \rightarrow(A \equiv C)$ holds by propositional logic, we may conclude as desired that $(A \equiv B) \rightarrow[(B \equiv C) \rightarrow(A \equiv C)]$.

$\mathbf{L 1}$ If $\mathcal{L}$ is transparent, then Ref and Imps entail $(A \equiv B) \rightarrow\left(C \equiv C_{[B / A]}\right)$.

Proof Assuming that $\mathcal{L}$ is transparent, the proof proceeds by induction on the complexity of $C \in \mathrm{wfs}(\mathbb{L})$. Of course, if $C \in \mathbb{L}$, then $(A \equiv B) \rightarrow\left(C \equiv C_{[B / A]}\right)$ holds by propositional logic if $A$ occurs in $C$, and $C \equiv C_{[B / A]}$ holds by Ref otherwise, where $(A \equiv B) \rightarrow\left(C \equiv C_{[B / A]}\right)$ follows by propositional logic.

Assume for induction that $(A \equiv B) \rightarrow\left(C \equiv C_{[B / A]}\right)$ holds whenever $\operatorname{comp}(C) \leqslant n$, and let $\operatorname{comp}(C)=n+1$. Assuming that $C=\mathcal{Q}^{n}(\vec{D})$, we may observe that for all $1 \leqslant i \leqslant n$ that $(A \equiv B) \rightarrow\left(D_{i} \equiv D_{i[B / A]}\right)$ follows by hypothesis, where $\left(D_{i} \equiv D_{i[B / A]}\right) \rightarrow\left[\mathcal{Q}^{n}(\vec{E}) \equiv \mathcal{Q}^{n}\left(\vec{E}_{\left[D_{i[B / A]} / D_{i}\right]}\right)\right]$ by the transparency of $\mathcal{L}$ for any $\vec{E}$. Assuming $A \equiv B$, it follows for all $1 \leqslant m \leqslant n$ that $\mathcal{Q}^{n}\left(\vec{D}_{\left[D_{1[B / A]} / D_{1}\right] \ldots\left[D_{m[B / A]} / D_{m}\right]}\right) \equiv \mathcal{Q}^{n}\left(\vec{D}_{\left[D_{1[B / A]} / D_{1}\right] \ldots\left[D_{m+1[B / A]} / D_{m+1}\right]}\right)$. Given that Ref and Imps, it follows by $\mathbf{P 1}$ that Trans holds, and so by $n-1$ applications of Trans, $\mathcal{Q}^{n}(\vec{D}) \equiv \mathcal{Q}^{n}\left(\vec{D}_{\left[D_{1[B / A]} / D_{1}\right] \ldots\left[D_{n[B / A]} / D_{n}\right]}\right)$. We may then observe that $\mathcal{Q}^{n}\left(\vec{D}_{\left[D_{1[B / A]} / D_{1}\right] \ldots\left[D_{n[B / A]} / D_{n}\right]}=\mathcal{Q}^{n}(\vec{D})_{[B / A]}\right.$, and so it follows by discharging our assumption that $(A \equiv B) \rightarrow\left(\mathcal{Q}^{n}(\vec{D}) \equiv \mathcal{Q}^{n}(\vec{D})_{[B / A]}\right)$. Since $C=\mathcal{Q}^{n}(\vec{D})$, we may conclude that $(A \equiv B) \rightarrow\left(C \equiv C_{[B / A]}\right)$.

P2 Assuming Ref and Imps, then $\mathcal{L}$ is transparent just in case $\mathbf{L} \mathbf{L}$ holds. 
Proof Assume Ref and Imps. Letting LL hold in $\mathcal{L}$ where $\mathcal{Q}$ is an operator in $\mathcal{L}$, it follows that $(A \equiv B) \rightarrow\left([\mathcal{Q}(\vec{O}) \equiv \mathcal{Q}(\vec{O})] \rightarrow\left[\mathcal{Q}(\vec{O}) \equiv \mathcal{Q}(\vec{O})_{(B / A)}\right]\right)$. However, given Ref, $\mathcal{Q}(\vec{O}) \equiv \mathcal{Q}(\vec{O})$, and so $(A \equiv B) \rightarrow\left[\mathcal{Q}(\vec{O}) \equiv \mathcal{Q}(\vec{O})_{(B / A)}\right]$. In particular, $(A \equiv B) \rightarrow\left[\mathcal{Q}(\vec{O}) \equiv \mathcal{Q}\left(\vec{O}_{(B / A)}\right)\right]$ as in Func. Generalising on $A, B, \vec{O}$, and $\mathcal{Q}$, we may conclude that $\mathcal{L}$ is transparent.

Assume instead that $\mathcal{L}$ is transparent. Letting $p \in \mathbb{L}$ where $p$ does not occur in $B$ or $C$, it follows by $\mathbf{L} 1$ that $(p \equiv B) \rightarrow\left(C_{(p / A)} \equiv C_{(p / A)[B / p]}\right)$. However, $C_{(p / A)[B / p]}=C_{(B / A)}$, and so $(p \equiv B) \rightarrow\left(C_{(p / A)} \equiv C_{(B / A)}\right)$. Thus it follows that, $(A \equiv B) \rightarrow\left(C \equiv C_{(B / A)}\right)$ and so $\mathbf{L L}$ follows by Imps.

Open Access This article is licensed under a Creative Commons Attribution 4.0 International License, which permits use, sharing, adaptation, distribution and reproduction in any medium or format, as long as you give appropriate credit to the original author(s) and the source, provide a link to the Creative Commons licence, and indicate if changes were made. The images or other third party material in this article are included in the article's Creative Commons licence, unless indicated otherwise in a credit line to the material. If material is not included in the article's Creative Commons licence and your intended use is not permitted by statutory regulation or exceeds the permitted use, you will need to obtain permission directly from the copyright holder. To view a copy of this licence, visit http://creativecommons.org/licenses/by/4.0/.

\section{References}

1. Anderson, A. R., Belnap, N. D., \& Dunn, J.M. (1976). Entailment: The Logic of Relevance and Necessity, 2nd edn. Vol. I. Princeton: Princeton University Press. ISBN 978-0-691-07192-3.

2. Angell, R. B. (1989). Deducibility, Entailment and Analytic Containment. In J. Norman, \& R. Sylvan (Eds.) Directions in Relevant Logic, Reason and Argument (pp. 119-143). Dordrecht: Springer Netherlands. ISBN 978-94-009-1005-8.

3. Bacon, A. (2019). Substitution structures. Journal of Philosophical Logic, 48, 1017-1075. ISSN 1573-0433. https://doi.org/10.1007/s10992-019-09505-z.

4. Berto, F. (2019). Simple hyperintensional belief revision. Erkenntnis, 84, 559-575. ISSN 1572-8420. https://doi.org/10.1007/s10670-018-9971-1.

5. Bou, F., \& Rivieccio, U. (2011). The logic of distributive bilattices. Logic. Journal of the IGPL, 19, 183-216. ISSN 1367-0751. https://doi.org/10.1093/jigpal/jzq041.

6. Brast-McKie, B. (2020). Towards a Logic of Essence and Ground. Ph.D. thesis, The University of Oxford.

7. Caie, M., Goodman, J., \& Lederman, H. (2019). Classical Opacity. Philosophy and Phenomenological Research n/a. ISSN 1933-1592. https://doi.org/10.1111/phpr.12587.

8. Correia, F. (2004). Semantics for Analytic Containment. Studia Logica, 77, 87-104. ISSN 1572-8730. https://doi.org/10.1023/B:STUD.0000034187.37935.24.

9. Correia, F. (2016). On the logic of factual equivalence. The Review of Symbolic Logic, 9, 103-122. ISSN 1755-0203, 1755-0211. https://doi.org/10.1017/S1755020315000258.

10. Correia, F., \& Skiles, A. (2019). Grounding, essence, and identity. Philosophy and Phenomenological research, 98, 642-670. ISSN 1933-1592. https://doi.org/10.1111/phpr.12468.

11. Dorr, C. (2016). To be $\mathrm{f}$ is to be g. Philosophical Perspectives, 30, 39-134. ISSN 1520-8583. https://doi.org/10.1111/phpe.12079.

12. Fine, K. (2015). Unified foundations for essence and ground. Journal of the American Philosophical Association, 1, 296-311. ISSN 2053-4477. https://doi.org/10.1017/apa.2014.26.

13. Fine, K. (2016). Angellic content. Journal of Philosophical Logic, 45, 199-226. ISSN 0022-3611, 1573-0433. https://doi.org/10.1007/s10992-015-9371-9.

14. Fine, K. (2017a). A theory of truthmaker content i: conjunction, Disjunction and Negation. Journal of Philosophical Logic, 46, 625-674. ISSN 0022-3611 1573-0433. https://doi.org/10.1007/s10992016-9413-y. 
15. Fine, K. (2017b). A theory of truthmaker content II: Subject-matter, common content, remainder and ground. Journal of Philosophical Logic, 46, 675-702. ISSN 0022-3611, 1573-0433. https://doi.org/10.1007/s10992-016-9419-5.

16. Fine, K. (2017c). Truthmaker Semantics. In A Companion to the Philosophy of Language (pp. 556577). New York: Wiley. ISBN 978-1-118-97209-0. https://doi.org/10.1002/9781118972090.ch22.

17. Fine, K. (2020). Yablo on Subject-Matter. Philosophical studies, 177, 129-171. ISSN 1573-0883. https://doi.org/10.1007/s11098-018-1183-7.

18. Fine, K., \& Jago, M. (2019). Logic for exact entailment. The review of symbolic logic, 12, 536-556. ISSN 1755-0203, 1755-0211. https://doi.org/10.1017/S1755020318000151.

19. Fitting, M. (1989a). Bilattices and the Semantics of Logic Programming.

20. Fitting, M. (1989b). Bilattices and the theory of truth. Journal of Philosophical Logic, 18, 225-256. ISSN 0022-3611, 1573-0433. https://doi.org/10.1007/BF00274066.

21. Fitting, M. (1990). Bilattices in logic programming. In Proceedings of the Twentieth International Symposium on Multiple-Valued Logic, (Vol. 1990 pp. 238-246). https://doi.org/10.1109/ISMVL.1990. 122627.

22. Fitting, M. (1991). Kleene's Logic, Generalized. Journal of Logic and Computation, 1, 797-810. ISSN 0955-792X, 1465-363X. https://doi.org/10.1093/logcom/1.6.797.

23. Fitting, M. (1994). Kleene's three valued logics and their children. Fundam. Inf., 20, 113-131. ISSN 0169-2968.

24. Fitting, M. (2002). Bilattices are nice things. Self-reference, 53-77.

25. Ginsberg, M. L. (1988). Multivalued logics: a uniform approach to inference in artificial intelligence. Computational Intelegence, 4, 265-316.

26. Ginsberg, M. L. (1990). ISSN 0955-792X, 1465-363X. https://doi.org/10.1093/logcom/1.1.41. Journal of Logic and Computation, 1, 41-69.

27. Hawke, P. (2018). Theories of aboutness. Australasian. Journal of Philosophy, 96, 697-723. ISSN 0004-8402. https://doi.org/10.1080/00048402.2017.1388826.

28. Heim, I. (1990). E-Type Pronouns and donkey anaphora. Linguistics and philosophy, 13, 137-177. ISSN 1573-0549. https://doi.org/10.1007/BF00630732.

29. Kratzer, A. (1989). An investigation of the lumps of thought. Linguistics and philosophy, 12, 607-653. ISSN 1573-0549. https://doi.org/10.1007/BF00627775.

30. Kratzer, A. (1998). Scope or Pseudoscope? Are There Wide-Scope Indefinites?. In S. Rothstein (Ed.) Events and Grammar, Studies in Linguistics and Philosophy (pp. 163-196). Dordrecht: Springer Netherlands. ISBN 978-94-011-3969-4.

31. Kratzer, A. (2002). Facts: Particulars or Information Units?. Linguistics and Philosophy, 25, 655-670. ISSN 1573-0549. https://doi.org/10.1023/A:1020807615085.

32. Lewis, D. (1988a). Relevant implication. Theoria, 54, 161-174. ISSN 1755-2567. https://doi.org/10. 1111/j.1755-2567.1988.tb00716.x.

33. Lewis, D. (1988b). Statements partly about observation. Philosophical papers, 17, 1-31. ISSN 05568641. https://doi.org/10.1080/05568648809506282.

34. Parry, W. T. (1989). Analytic Implication; Its History, Justification and Varietiess. In J. Norman, \& R. Sylvan (Eds.) Directions in Relevant Logic, Reason and Argument (pp. 101-118). Dordrecht: Springer Netherlands. ISBN 978-94-009-1005-8. https://doi.org/10.1007/978-94-009-1005-87.

35. Perry, J. (1989). Possible Worlds and Subject Matter. In The Problem of the Essential Indexical and Other Essays (pp. 145-60). Palo Alto: CSLI Publications.

36. Rayo, A. (2013). The construction of logical space. Oxford: Oxford university press. ISBN 9780199662623, hbk.

37. von Fintel, K., \& Partee, B. H. (2002). A Minimal Theory of Adverbial Quantification. In H. Kamp (Ed.) Context-Dependence in the Analysis of Linguistic Meaning, volume 11 of Current Research in the Semantics/Pragmatics Interface (pp. 137-175). Amsterdam: Brill. ISBN 978-0-08043694-4.

38. Yablo, S. (2014). Aboutness. Berlin: Princeton University Press. ISBN 978-1-4008-4598-9.

Publisher's Note Springer Nature remains neutral with regard to jurisdictional claims in published maps and institutional affiliations. 